\title{
Anabases
}

ANABASES Traditions et réceptions de l'Antiquité

$28 \mid 2018$

Varia

\section{Tucidide e il Fascismo. Su una pagina dimenticata de La Rivoluzione Liberale di Piero Gobetti}

Thucydides and Fascism. Some Remarks on a Forgotten Page of Piero Gobetti's La Rivoluzione Liberale

Luca lori

\section{CpenEdition}

\section{Journals}

Edizione digitale

URL: http://journals.openedition.org/anabases/7518

DOI: 10.4000/anabases.7518

ISSN: 2256-9421

\section{Editore}

E.R.A.S.M.E.

Edizione cartacea

Data di pubblicazione: 9 novembre 2018

Paginazione: 47-79

ISSN: 1774-4296

Notizia bibliografica digitale

Luca Iori, «Tucidide e il Fascismo. Su una pagina dimenticata de La Rivoluzione Liberale di Piero Gobetti», Anabases [Online], 28 | 2018, Messo online il 09 novembre 2020, consultato il 20 janvier 2021. URL: http://journals.openedition.org/anabases/7518; DOI: https://doi.org/10.4000/anabases 7518

(c) Anabases 
Anabases 28 (2018), p. 47-79.

\section{Tucidide e il Fascismo. Su una pagina dimenticata de La Rivoluzione Liberale di Piero Gobetti}

Luca Iori

l 18 novembre 1924, cinque mesi dopo l'omicidio di Giacomo Matteotti e in un clima di crescente fibrillazione politica, Piero Gobetti riservò le prime colonne de La Rivoluzione Liberale (anno III, n. 43) a un breve e sorprendente articolo intitolato "Tucidide e il fascismo». Il pezzo, che venne pubblicato senza firma, raccoglieva una serie di excerpta tucididei in traduzione italiana tratti dal terzo e dall'ottavo libro delle Storie: III, 82-83 e VIII, 63, 65-66'. Anticipati da brevi titoletti provocatori (“La Marcia su Roma e i salvatori della Patria», "Gli assassini», «I "Ras” e l'amministrazione», etc.), i brani sviluppavano un malizioso accostamento tra passato e presente, invitando i lettori a interpretare in maniera polemica il quadro politico dell'epoca: facendo dialogare l'attualità con le vicende del colpo di stato oligarchico ateniese (411 a.C.) e con la sanguinosa guerra civile di Corcira (427-425 a.C.), l'articolo ambiva a recuperare la memoria classica in chiave schiettamente anti-autoritaria e sviluppava una lucida analisi delle logiche criminali del regime mussoliniano, ormai prossimo a imboccare la strada dell'aperta dittatura. Così, a dispetto del suo carattere apparentemente compilativo e della sua modesta estensione, la prosa pubblicata da Gobetti si faceva testimone di un riuso politico dell'opera tucididea che si segnala ancora oggi per l'approccio spregiudicato alla storia antica.

Tali considerazioni, affiorate in maniera alquanto sporadica nella pur vasta bibliografia gobettiana ${ }^{2}$, non sono tuttavia valse a sviluppare un esame

1 Una trascrizione integrale dell'articolo è ripubblicata in Appendice (infra p. 77-79).

2 Cf. e.g. Opere complete di Piero Gobetti, I, Scritti politici, a cura di P. Spriano, Torino, 
sufficientemente approfondito di questo singolare esperimento di lotta politica attraverso i classici. Proprio per la sua natura di adespoto, infatti, il “corsivo tucidideo» è rimasto sempre escluso dalle raccolte di scritti pubblicate sotto il nome dell'intellettuale piemontese, ottenendo così, presso la critica, una scarsissima visibilità. Nell'intento di sottrarlo a questo oscuro e immeritato destino, varrà dunque la pena di riproporne un più dettagliato inquadramento nel contesto del laboratorio gobettiano, discutendo l'articolo dapprima in relazione alla linea editoriale assunta dalla rivista nel convulso tornante politico del 1924 (§.1) e, successivamente, illuminando la complessa tecnica centonaria che ne fu alle spalle e che ne determinò, in ultima istanza, la fisionomia complessiva (§.2). Muovendoci in questa duplice prospettiva - storica e formale - sarà quindi possibile acquisire nuovi elementi per riesaminare, nella seconda parte del contributo (§.3-4), i presupposti teorici di questa audace prosa di ispirazione classicistica maturata negli ambienti più radicali del liberalismo torinese e del tutto estranea alle pulsioni antidemocratiche che alimentarono la coeva riappropriazione del passato romano da parte del fascismo ${ }^{3}$.

\section{«ll lettore deve leggere tra le righe»: Gobetti e la lotta politica del 1924.}

“Mi si riferisce che noto Gobetti sia stato recentemente a Parigi e che oggi sia in Sicilia. Prego informarmi e vigilare per rendere nuovamente difficile vita questo insulso oppositore di Governo e fascismo. Mussolini ${ }^{4}$ ). Il telegramma, trasmesso il $1^{\circ}$ giugno 1924 al Prefetto di Torino, descrive bene il clima di controlli e rappresaglie in cui l'editore piemontese - da tempo sorvegliato dalle autorità fasciste - era costretto a vivere ${ }^{5}$. Già nel 1923, su ordine di Mussolini, Gobetti

1960, p. 800; F. Brioschi, L’azione politico-culturale di Piero Gobetti, Milano, 1974, p. 150-151; P. Spriano, Gramsci e Gobetti. Introduzione alla vita e alle opere, Torino, 1977, p. 128; S. Festa, Gobetti, Assisi, 1980, p. 125; P. Meaglia, «Stato ed economia in Gobetti», Annali della Fondazione Luigi Einaudi 16 (1982), p. 410.

3 Su questi temi vd. spec. L. Canfora, Ideologie del classicismo, Torino, 1980, p. 57-132 e ID., Le vie del classicismo, Roma-Bari, 1989, p. 253-277, ai quali si rimanda, qui e di seguito, per una sintetica e ormai canonica discussione del classicismo come «matrice culturale» e ideologica del fascismo.

4 R. De Felice, Intellettuali di fronte al fascismo. Saggi e note documentarie, Roma, 1985, p. 250.

5 Per un sintetico ed efficace profilo biografico di Gobetti nei primi anni Venti cf. C. Malandrino, s.v. "Gobetti», Dizionario Biografico degli Italiani, LVII, 2001, p. 495ss. Sul contesto politico-culturale torinese, vd. A. D'Orsi, “La cultura e i gruppi intellettuali', in Storia di Torino, VIII, a cura di N. Tranfaglia, Dalla Grande Guerra 
aveva subìto perquisizioni e sequestri di materiali, trascorrendo due brevi periodi nelle carceri torinesi: la prima volta, nel mese di febbraio, accusato di cintelligenza coi comunisti ${ }^{6}$ »; la seconda, il 29 maggio, con l'imputazione di aver collaborato - in veste di critico teatrale - al quotidiano L'Ordine Nuovo, diretto da Antonio Gramsci. Pochi mesi dopo, il 22 febbraio 1924, il Duce in persona, durante un incontro a Palazzo Chigi, avrebbe preteso per il «seccante» Gobetti una "severa lezione fascista", poi rimandata per alcune divisioni sorte in seno allo squadrismo torinese ${ }^{7}$.

Questa crescente aggressività, resa evidente dall'incrudelire delle punizioni prospettate, derivava dalla notevole visibilità assunta dalle iniziative gobettiane. Dall'inizio del '24, infatti, l'intellettuale torinese aveva manifestato una linea di opposizione sempre più efficace nei confronti del governo, rafforzando il legame tra le uscite de La Rivoluzione liberale e la sua casa editrice - la Piero Gobetti Editore - che in vista delle elezioni politiche del 6 aprile aveva sfornato scritti dal forte impegno pubblico ${ }^{8}$. A questi aveva fatto seguito la ristampa, presso Cappelli, di alcuni corsivi gobettiani che abbozzavano un programma di

alla Liberazione (1915-1945), Torino, 1998, p. 499-622, e ID., La cultura a Torino tra le due guerre, Torino, 2000.

6 L'ordine d'arresto venne trasmesso direttamente da Mussolini al Prefetto di Torino il 6 febbraio 1923: “Ordinole perquisire immediatamente redazione amministrazione giornale 'RIVOLUZIONE LIBERALE' sequestrando schedarî abbonati corrispondenza libri amministrativi-stop Contemporaneamente procederà arresto nominato Pietro Gobetti e redattori provvedendo a denunciarlo autorità giudiziaria per intelligenza coi comunisti sovversivi-stop» (De Felice, Intellettuali, p. 252). Un altro telegramma dell'8 marzo documenta la profonda avversione di Mussolini nei confronti del periodico gobettiano: “Giornale 'RIVOLUZIONE LIBERALE' è stato uno dei nemici più perfidi sebbene innocui attuale Governo stop Tutte le canaglie espulse vita politica italiana vi si sono date convegno" (ivi, p. 253).

7 L'episodio è descritto da Guido Narbona, tenente della Milizia fascista, in una lettera datata del 24 novembre 1924 (a cura di G. Rossini, vd. Il delitto Matteotti tra il Viminale e l'Aventino. Dagli atti del processo De Bono davanti all'Alta Corte di Giustizia, Bologna, 1966, p. 264-266).

$8 \quad$ E.g. Una battaglia liberale di Giovanni Amendola, Popolarismo e fascismo di Luigi Sturzo. Sulle strategie editoriali della casa gobettiana nel 1924, vd. spec. M.A. FвавоттA, Gobetti. L'editore giovane, Bologna, 1988, p. 109-140, con E. Alessandrone Perona, "Gobetti editore (Appunti)", in Piero Gobetti e il suo tempo, Torino, 1976, p. 33-51; M. Accame Lanzillotta, Le edizioni e i tipografi di Piero Gobetti. Studio di bibliografia storica, Firenze, 1980; E. Alessandrone Perona, “Gobetti editore: dal 'modello vociano' all'editore ideale», in P. Soddu (a cura di), Giulio Einaudi nell'editoria di cultura nel Novecento italiano, Firenze, 2015, p. 20ss. 
lotta politica contro il fascismo9. L'esito negativo delle urne, favorito dai brogli squadristici e dai meccanismi truffaldini della legge Acerbo, non valse ad arrestare le iniziative di Piero, che a maggio, per rilanciare l'azione dei gruppi antifascisti, intraprese una serie di viaggi a Parigi e in Sicilia, attirando proprio con questi spostamenti l'attenzione di Mussolini, il quale, con il telegramma del $1^{\circ}$ giugno, pretese «nuovamente» un intervento contro "questo insulso oppositore [del] fascismo". L'ordine, trasmesso al prefetto, venne puntualmente eseguito alle 9.30 del 9 giugno, quando un manipolo di agenti senza regolare mandato fece irruzione nell'abitazione-redazione di Gobetti, sottoponendolo a una violenta aggressione fisica e sequestrandone l'intera corrispondenza politica.

Nonostante la rappresaglia, l'editore non si lasciò intimidire e a partire dal giorno successivo - quello dell'inattesa e traumatica scomparsa di Giacomo Matteotti - la sua attività segnò un ulteriore cambio di passo. Intuendo la natura eversiva dell'azione, La Rivoluzione Liberale pubblicò vari corsivi di denuncia che culminarono nell'uscita di un numero monografico dedicato al deputato socialista $\left(1^{\circ}\right.$ luglio 1924), in cui un lungo scritto gobettiano - poi ripubblicato con enorme successo in forma di volumetto - ripercorreva con toni agiografici la carriera pubblica e le battaglie di Matteotti ${ }^{10}$. Parallelamente, la rivista si presentò all'attenzione nazionale come entità politica: il 18 giugno, a Torino, il cgruppo della 'Rivoluzione Liberale'» prese parte ad un'assemblea programmatica di formazioni antifasciste e l'8 luglio Gobetti lanciò dalle colonne del suo giornale un vigoroso appello alla costituzione di altri «Gruppi» in tutta la penisola, ottenendo buoni riscontri a Roma, Milano e Napoli"'.

Sulla spinta di queste e altre campagne di mobilitazione, il 10 luglio 1924 il governo Mussolini decise di promulgare un decreto legge che introduceva una serie di norme fortemente restrittive nei confronti della stampa ${ }^{12}$. Il controllo su quotidiani e periodici si fece più pressante e lo spazio per la critica angusto: i prefetti ottennero ampia facoltà di censurare, sequestrare e sospendere tutti i giornali che fossero ritenuti responsabili di turbare l'ordine pubblico e anche La Rivoluzione Liberale, messa di fronte alla concreta possibilità di interrompere le proprie pubblicazioni, dovette adeguarsi alle nuove regole. Il giornale decise

9 P. Gobetri, La Rivoluzione Liberale. Saggio sulla lotta politica in Italia, Bologna, 1924 (successivamente citato secondo l'edizione di Ersilia Alessandrone Perona [20084], vd. infra n. 34).

10 P. Goвetтi, “Matteotti», La Rivoluzione Liberale III (1924), n. 27, p. 105-107. La numerazione delle pagine della rivista - d'ora in poi $R L$ nelle citazioni - segue la riproduzione anastatica di La Rivoluzione Liberale. Rivista storica settimanale di politica (I [1922], n. 1 - IV [1925], n. 40), Parma, 1967.

11 RL III (1924), n. 28, p. 110 e n. 31, p. 128.

12 Regio D.L. del 10 luglio 1924, n. 1081. 
tuttavia di non abdicare alla propria missione e adottò nuove e più ingegnose strategie editoriali. Tra queste, prese forma l'idea di rilanciare la lotta politica attraverso la riappropriazione, maliziosa e irriverente, della memoria storica.

Se infatti corsivi e articoli di attualità rischiavano di subire ampie limitazioni, più difficile sarebbe stato per le autorità condannare le parole di autori come Tucidide, Machiavelli o Massimo D’Azeglio, che venivano quotidianamente studiati nelle scuole e nelle università del regno. Allo stesso modo, riproporre in chiave critica vivaci affreschi storici o profili di grandi personalità del passato come Giulio Cesare o Lorenzo de' Medici, permetteva di commentare, in maniera obliqua ma del tutto efficace, la realtà contemporanea sfruttando il potere evocativo dell'analogia storica. Per rendere efficace questa strategia era tuttavia necessario metterne al corrente i lettori, i quali vennero puntualmente avvertiti tramite il seguente avviso, stampato in grassetto sulla testata di tutti i numeri del mese di luglio: “In regime di stampa imbavagliata il vero articolista è il lettore: egli deve leggere tra le righe ${ }^{13}$ ). E nell'uscita del giorno 22 (anno III, n. 30), un articolo di fondo, firmato La Rivoluzione Liberale, rese ancor più esplicito il monito:

Mantenere un periodico libero in tempi avventurosi deve significare affidarsi all'intelligenza del pubblico, rinunciare al pubblico facile e superficiale. Noi abbiamo la fortuna, che non ha nessun altro giornale, di parlare a un pubblico piccolo ma scelto. Possiamo contare sulle risonanze, sul commento, su una specie di intesa nelle premesse. [...] Impegniamo dunque il lettore alla gara singolare: e il premio sia per chi saprà trovar significati più arguti ai sottintesi, leggere e scrivere più pungentemente tra le righe, ricamare malignità nelle cose più innocenti, interpretare da buoni moderni la storia antica. Sarcasmi, ironie, malizie valgano dunque, poiché tali sono i tempi, in luogo di una professione di fede ${ }^{14}$.

Questo genere di giochi retrospettivi non era in realtà inedito per la rivista: già nel novembre '22, una libera traduzione di Archiloco [= fr. $115 \mathrm{West}^{2}$ ] aveva ironizzato sul potere acquisito da Mussolini dopo la Marcia su Roma, favorendo la sovrapposizione della figura del Duce a quella del tiranno Leofilo, trasfigurato, per l'occasione, nelle fattezze di un intraprendente Figaro («Or Leòfilo comanda, alto e basso Leofilo fa | tutti pendon dalla bocca di Leofilo | è Leofilo il factotum in città ${ }^{15}$ ); un anno dopo, nell'ottobre 1923, un'antologia di brani di Machiavelli (Arte della Guerra, libro I) denunciava le violenze delle milizie volontarie

13 RL III (1924), nn. 29, 30, 31.

14 [P. Gobetti] «La nostra difesa», $R L$ III (1924), n. 30, p. 117. Corsivi miei.

15 Archiloco, [“Leofilo»] RL I (1922), n. 32, p. 119 [trad. di Ettore Romagnoli]. L'originale

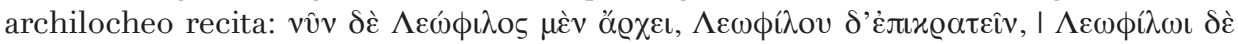

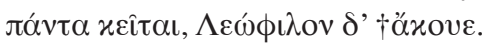


fasciste $^{16}$, mentre nel maggio 1924 un secondo centone machiavelliano - questa volta ricavato dai Discorsi - celebrava la «fede nelle forze popolari» e il loro ruolo progressivo nella vita dello stato ${ }^{17}$. Fu tuttavia con l'introduzione della nuova legge sulla stampa nel luglio '24 che il ricorso a tali espedienti divenne sistematico, e così, tra l'estate e l'autunno di quell'anno, iniziarono a moltiplicarsi articoli che rievocavano eventi passati per ridiscutere in maniera critica e mordace l'ascesa violenta del movimento mussoliniano.

Non potendo ripercorrere per intero la trama polemica di queste prose, possiamo comunque notare che l'attenzione di Gobetti e dei suoi collaboratori andò appuntandosi su tre periodi tradizionalmente considerati decisivi nel processo di formazione della coscienza nazionale: la Roma repubblicana, le vicende dello stato fiorentino dal Tre al Cinquecento e i moti risorgimentali. Muovendo lungo questi assi privilegiati, e alternando antologie d'autore, stralci di saggi e pungenti corsivi, gli articolisti sfruttavano l'aura di esemplarità comunemente attribuita agli avvenimenti storici per commentare l'attualità ${ }^{18}$. Mussolini veniva così descritto tramite le "vite parallele" di Catilina e Cesare - «non un grande uomo di Stato, ma il

16 N. Machiavelli, “Consigli machiavellici ai nepoti», $R L$ II (1923), n. 31, p. 127.

17 N. Machiavelli, “Commento a un preludio», $R L$ III (1924), n. 20, p. 77. L'articolo uscì in risposta al celebre “Preludio a Machiavelli» pubblicato da Mussolini su Gerarchia il 30 aprile 1924. Sulla prosa mussoliniana - anticipatrice per molti aspetti della dottrina dello Stato fascista - e sulle pronte repliche di Gobetti (su La Rivoluzione Liberale) e Matteotti (su English Life), vd. ora L. Mitarotondo, Un 'Preludio' a Machiavelli. Letture e interpretazionifra Mussolini e Gramsci, Torino, 2016.

18 Segnaliamo tra gli altri, in ordine di apparizione sulla rivista: 1) F.S. NitTi, “Il colpo di stato", RL III (1924), n. 28, p. 111-112 - brani tratti da ID. Sui moti di Napoli del 1820, Firenze, 1897; 2) G. Ansaldo, “I fascisti dissidenti», RL III (1924), n. 29, p. 113, che confronta la biografia di Lorenzino de' Medici a quella di Cesare Forni; 3) L.C. Farini, “Normalizzazione», $R L$ III (1924), n. 31, p. 125, raccolta di brani sui moti romagnoli del 1831 e del 1845 tratta da ID., Manifesto di Rimini, Rimini, 1845 e iD., Lo Stato Romano dall'anno 1815 all'anno 1850, Firenze, 1850; 4) N. Machiavelli, “Il Duca d'Atene», RL III (1924), n. 31, p. 125, citazione di ID., Istorie fiorentine, II, 36, 4-7; 5) G. Ferrero, “La dittatura di Cesare», $R L$ III (1924), n. 32, p. 132, antologia sul governo cesariano post 46 a.C. da id., Grandezza e decadenza di Roma, II, Giulio Cesare, Milano, 1902; 6) SAllustio, "Catilinaria», $R L$ III (1924), n. 38, p. 153, raccolta di passi, in traduzione italiana, da Sall. Catil. 5.1-2, 4-6; 14.1-3; 21.1-2, 4; 25.1-5; 37.4-7; 52.5-6, 7) M. D’Azeglio, “Degli ultimi casi di... Romagna», $R L$ III (1924), n. 42, p. 171, antologia sui moti romagnoli del 1845 da ID., Degli ultimi casi di Romagna, s.n., 1846; 8) Tucidide, "Tucidide e il fascismo", RL III (1924), n. 43, p. 173, cf. supra; 9) K.E. Suckert, “Del perfetto tiranno», RL III (1924), n. 44, p. 179, epistola ficta di Lorenzo de' Medici a Mussolini; 10) A. Cavalli, "Commemorazione anticipata del Duce», $R L$ III (1924), n. 44, p. 179, che accosta la figura di Mussolini al capitano di ventura romagnolo Muzio Attendolo Sforza. 
più gran demagogo della storia ${ }^{19}$ »; gli squadristi erano identificati con i Catilinari e i Sanfedisti romagnoli ${ }^{20}$, e le politiche assistenzialiste del Duce venivano assimilate alle regalie del Duca di Atene alla plebe fiorentina ${ }^{21}$. Dall'altro lato, sul versante dei potenziali oppositori, la parabola del tirannicida Lorenzino de' Medici spiegava l'inaspettato dissenso del fedelissimo Cesare Forni, prima organico al potere centrale, poi ribelle perché da esso marginalizzato ${ }^{22}$; la remissività di Vittorio Emanuele III si rispecchiava nell'acquiescenza di Ferdinando I di Borbone, messo alle strette dai carbonari napoletani ${ }^{23}$, e l'aspirazione alla lotta clandestina, da più parti invocata dopo l'omicidio Matteotti, veniva confutata attraverso i moniti di Massimo D’Azeglio, che, commentando i fatti di Romagna del 1845, aveva criticato le congiure segrete a favore di una lotta politica condotta alla luce del sole ${ }^{24}$.

“Tucidide e il fascismo», pubblicato il 18 novembre 1924, apparve al termine di questa lunga sequenza di prose e ne mutuò, come vedremo, i principali temi. Proprio per tale esaustività, l'articolo si inquadrava alla perfezione nel frenetico contesto politico dell'autunno '24, segnato dal tentativo delle opposizioni di infliggere la spallata decisiva al governo Mussolini. Il 12 novembre, infatti, la Camera aveva riaperto i battenti dopo oltre quattro mesi di inattività e l'Aventino aveva rilanciato l'idea di un governo di transizione che potesse indire a breve nuove elezioni. Il 15 novembre, i liberali di Giovanni Giolitti si erano sfilati dalla maggioranza, votando contro la fiducia a Mussolini e minacciando di condizionare altri settori dell'emiciclo. Due settimane più tardi, il 30 novembre, gli aventiniani promossero a Milano un nuovo raduno di protesta e poco dopo, il 5 dicembre, il Senato, pur votando a favore dell'esecutivo, vide crescere ancora il numero degli astenuti e dei contrari.

Nel pieno di questa escalation, il "corsivo tucidideo" entrava quiridi a far parte di una vigorosa campagna anti-regime finalizzata ad alienare il consenso parlamentare e popolare al governo. Come sappiamo, lo sviluppo degli eventi fu molto diverso dalle aspettative aventiniane e il 3 gennaio 1925 Mussolini assunse pubblicamente la responsabilità del delitto Matteotti, imprimendo una svolta decisiva verso la dittatura, concretizzatasi nel biennio successivo attraverso una serie di leggi e decreti che smantellarono quanto restava dell'impalcatura costituzionale dello stato post-unitario. Tuttavia, poche settimane prima, quando ancora la prospettiva di una stretta autoritaria appariva evitabile, Gobetti e i suoi

19 Sallustio, “Catilinaria», p. 153; Ferrero, “La dittatura di Cesare», p. 132.

20 Sallustio, “Catilinaria», p. 153; L.C. Farini, «Normalizzazione», p. 125.

21 N. Machiavelli, “Il Duca d'Atene», p. 125.

22 G. Ansaldo, “I fascisti dissidenti», p. 113.

23 F.S. NitTi, «Il colpo di stato», p. 111.

24 M. D’Azeglio, «Degli ultimi casi di... Romagna», p. 171. 
collaboratori si erano rivolti a Tucidide per denunciare l'illegittimità dell'autorità fascista, promuovendo, attraverso le parole dello storico, una presa di coscienza netta e intransigente nei confronti degli abusi del potere mussoliniano. Ma quali erano i passi tucididei che venivano mobilitati contro il regime fascista? E in che modo essi venivano adattati alle esigenze della lotta politica? Solo un esame ravvicinato dell'antologia può fornire le risposte a questi interrogativi.

\section{Anatomia di un centone: Tucidide tra la Marcia su Roma e l'affaire Matteotti.}

Come abbiamo ricordato, “Tucidide e il fascismo» raccoglieva dodici brani tratti da due celebri e distinte sezioni delle Storie: l'una, Thuc. III, 82-83, commentava la fase più cruenta della stasis di Corcira (427 a.C.); l'altra, Th. VIII, 63, 65-66, ripercorreva le prime convulse tappe del putsch oligarchico di Atene (411 a.C.). Ogni excerptum, tradotto in lingua italiana, era preceduto da un titoletto provocatorio, che indirizzava i lettori verso un malizioso raffronto tra i fatti della Guerra del Peloponneso e la scottante attualità dell'Italia fascista (fig. 1). Se la scelta dei temi e dei brani tucididei esprimeva l'ambizione di calare gli avvenimenti del '24 all'interno di una griglia interpretativa di sicuro spessore teorico, la distribuzione dei loci - concatenati secondo un ordine inedito - tracciava una progressione argomentativa del tutto originale, che merita di essere ripercorsa nel dettaglio.

L'esordio dell'antologia, affidato ad una coppia di brani relativi al coup d'état ateniese, ridiscuteva le fasi più traumatiche dell'ascesa mussoliniana: il primo passo dipingeva il golpe del '22 come un'iniziativa di stampo reazionario, che sopprimeva la libertà democratica a favore di un governo oligarchico e conservatore (“La Marcia su Roma e i salvatori della Patria» = Th. VIII, 63, 3 + Th. VIII, 65, 3 - 66, 1); il secondo excerptum rievocava l'uccisione del democratico Androcle, alludendo scopertamente all'affaire Matteotti (“Gli assassini» = Th. VIII, 65, 2):

Fu verso quell'epoca che la democrazia venne abolita in Atene [...] Da più tempo gli oligarchi avevano fatto circolare la voce che tutti i diritti spettavano unicamente agli uomini di guerra ed ai soli cittadini capaci di servire la città con la persona e con gli averi. Non si trattava in realtà che di un tranello teso alla moltitudine, poiché era chiaro che soltanto i fautori del colpo di Stato si sarebbero avvantaggiati del potere.

Si principiò con l'assassinio di Androclo, uno dei capi più in vista della democrazia. Quindi tutta la parte popolare fu presa d'assalto con un crescendo di uccisioni sistematiche.

Così introdotto, il centone proseguiva con un secondo gruppo di brani ancora tratti dall'ottavo libro - che registravano le reazioni delle parti in causa, stigmatizzando l'impunito insolentire della fazione oligarco-fascista ( La paura» = 


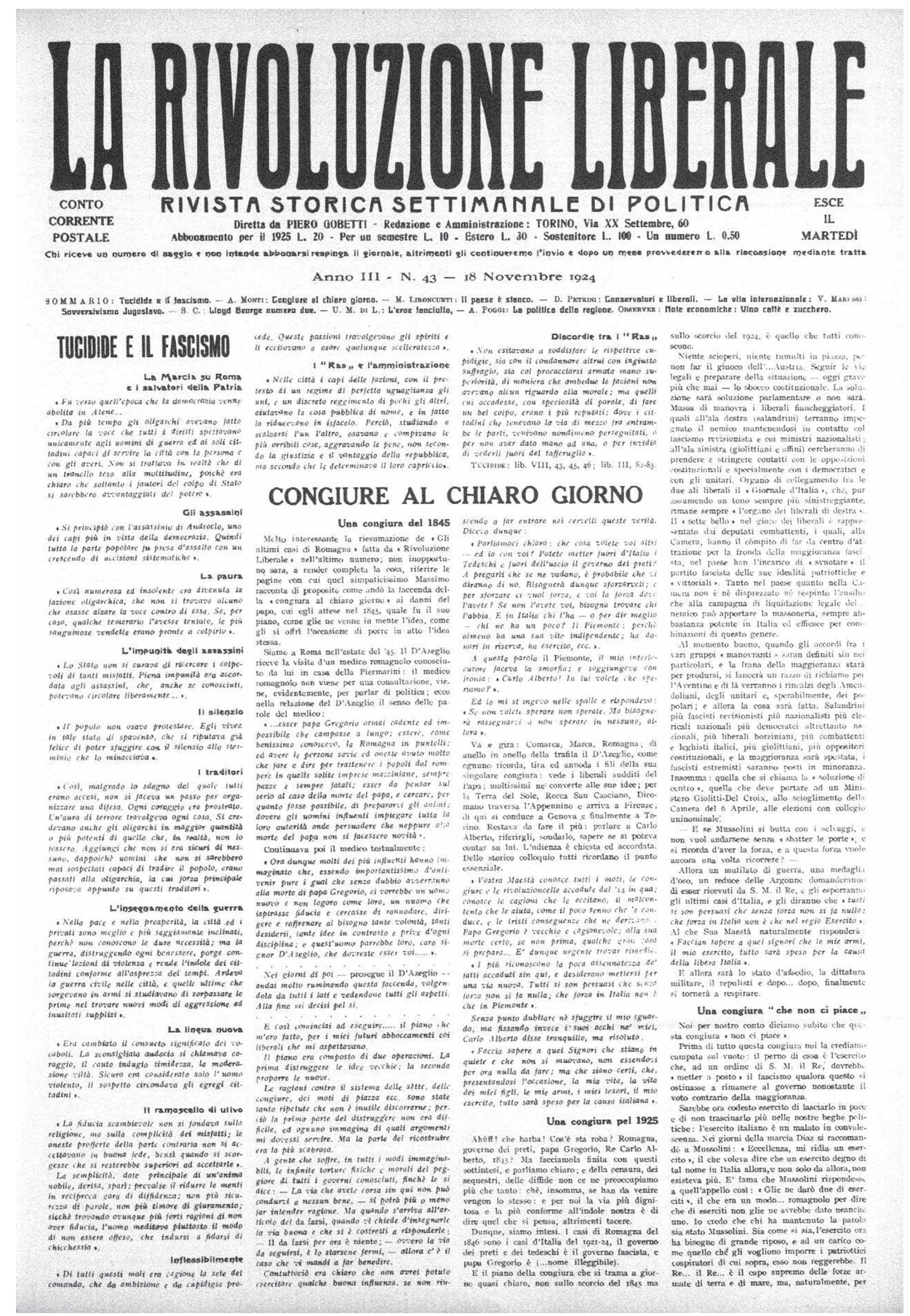

Fig. 1 “Tucidide e il fascismo», La Rivoluzione Liberale III (1924), n. 43, p. 173. 
Th. VIII, 66, 2; “L'impunità degli assassini» = Th. VIII, 66, 2), la passività del popolo (“Il silenzio» = Th. VIII, 66, 2) e lo spaesamento dei democratici ( I traditori» = Th. VIII, 66, 4 + VIII, 66, 3 + VIII, 66, 5):

Così numerosa ed insolente era divenuta la fazione oligarchica, che non si trovava alcuno che osasse alzare la voce contro di essa. Se, per caso, qualche temerario l'avesse tentato, le più sanguinose vendette erano pronte a colpirlo.

Lo Stato non si curava di ricercare i colpevoli di tanti misfatti. Piena impunità era accordata agli assassini, che, anche se conosciuti, potevano circolare liberamente...

Il popolo non osava protestare. Egli vivea in tale stato di spavento, che si riputava già felice di poter sfuggire con il silenzio allo sterminio che lo minacciava.

Così, malgrado lo sdegno del quale tutti erano accesi, non si faceva un passo per organizzare una difesa. Ogni coraggio era prostrato. Un'aura di terrore travolgeva ogni cosa. Si credevano anche gli oligarchi in maggior quantità o più potenti di quello che, in realtà, non lo fossero. Aggiungi che non si era sicuri di nessuno, dappoichè uomini che non si sarebbero mai sospettati capaci di tradire il popolo, erano passati alla oligarchia, la cui forza principale riposava appunto su questi traditori.

Una terza serie di excerpta - questa volta di derivazione corcirese - descriveva il degrado morale e civile seguito agli atti sediziosi appena descritti. Ne emergeva un corpo sociale imbarbarito dalle violenze e dagli effetti di una propaganda speciosa (“L'insegnamento della guerra» = Th. III, 82, 2-3; “La lingua nuova» = Th. III, 82, 4 + Th. III, 82, 5), che fomentava la diffidenza fra i cittadini, rendendo insicuro ogni accordo tra le parti (“Il ramoscello di ulivo» = Th. III, 82, 6-7 + Th. III, 83, 1-2). "Di tutti questi mali - si osservava - era cagione la sete del comando», che ctravolgeva gli spiriti [degli oligarchici], eccitandoli a osare qualunque scelleratezza» (“Inflessibilmente» = Th. III, 82, 8):

Nella pace e nella prosperità, la città ed i privati sono meglio e più saggiamente inclinati, perché non conoscono le dure necessità; ma la guerra, distruggendo ogni benessere, porge continue lezioni di violenza e rende l'indole dei cittadini conforme all'asprezza dei tempi. Ardeva la guerra civile nelle città, e quelle ultime che sorgevano in armi si studiavano di sorpassare le prime nel trovare nuovi modi di aggressione ed inusitati supplizi.

Era cambiato il consueto significato dei vocaboli. La sconsigliata audacia si chiamava coraggio, il cauto indugio timidezza, la moderazione viltà. Sicuro era considerato solo l’uomo violento, il sospetto circondava gli egregi cittadini.

La fiducia scambievole non si fondava sulla religione, ma sulla complicità dei misfatti; le oneste profferte della parte contraria non si accettavano in buona fede, bensì quando si scorgesse che si resterebbe superiori ad accettarle. La semplicità, dote principale di un'anima nobile, derisa, sparì; prevalse il ridurre le menti in reciproca 
gara di diffidenza; non più sicurezza di parole, non più timore di giuramento; sicché trovando ovunque più forti ragioni di non aver fiducia, l'uomo meditava piuttosto il modo di non essere offeso, che indursi a fidarsi di chicchessia.

Di tutti questi mali era cagione la sete del comando, che da ambizione e da cupidigia procede. Queste passioni travolgevano gli spiriti e li eccitavano a osare qualunque scelleratezza.

Coerentemente con questo sviluppo, l'antologia si chiudeva con un'altra coppia di passi corciresi che illustrava la sfrenatezza dei fiduciari di Mussolini (“I "Ras" e l'amministrazione» = Th. III, 82, 8; “Discordie tra i "Ras”» = Th. III, 82, 8), ormai liberi di dedicarsi a una selvaggia lotta per il potere e di accanirsi contro chi si rifiutava di fiancheggiarli:

Nelle città i capi delle fazioni, con il pretesto di un regime di perfetta uguaglianza gli uni, e un discreto reggimento di pochi gli altri, aiutavano la cosa pubblica di nome, e in fatto la riducevano in isfacelo. Perciò, studiando a scalzarsi l'un l'altro, osavano e compivano le più orribili cose, aggravando le pene, non secondo la giustizia e il vantaggio della repubblica, ma secondo che le determinava il loro capriccio.

Non esitavano a soddisfare le rispettive cupidigie, sia con il condannare altrui con ingiusto suffragio, sia col procacciarsi armata mano superiorità, di maniera che ambedue le fazioni non avevano alcun riguardo alla morale; ma quelli cui accadesse, con speciosità di parole, di fare un bel colpo, erano i più reputati; dove i cittadini che tenevano la via di mezzo fra entrambe le parti, venivano nondimeno perseguitati, o per non aver dato mano ad una, o per invidia di vederli fuori del tafferuglio.

Così impostato, lo scheletro argomentativo dell'antologia finiva insomma per denunciare i presupposti criminali del potere fascista, valutandone l'impatto traumatico sulla società e sulle regole della convivenza democratica. La brutale efficacia con cui l'articolo descriveva la vita civile della penisola non si esauriva però negli schemi astratti dell'analisi politica, ma si traduceva in un concreto monito alla nazione, che veniva indirettamente chiamata a esprimere un'opposizione ferma e intransigente, secondo quanto propugnato da tempo dalla rivista gobettiana. Non a caso, pochi giorni dopo l'uscita di “Tucidide e il fascismo», lo stesso Gobetti, gettando un occhio all'imponente manifestazione milanese del 30 novembre, descriveva in questi termini l'auspicata convergenza con gli aventiniani: “In tre mesi la nostra battaglia è vinta su tutta la linea: la tesi di Rivoluzione Liberale è oggi accettata dalle opposizioni, i discorsi di Amendola e di Turati a Milano parlarono almeno altrettanto chiaro come i nostri articoli sul tema dell'intransigenza ${ }^{25}$ ».

Se dunque è legittimo identificare nel doppio registro della denuncia e dell'organizzazione del dissenso le finalità ultime del centone tucidideo, restano 
da approfondire i criteri che orientarono la rifunzionalizzazione del materiale antico in chiave contemporanea. In particolare, merita di essere considerata la notevole qualità della traduzione pubblicata da Gobetti. Quest'ultima offriva una resa sostanzialmente indipendente rispetto a quelle comunemente circolanti fatta eccezione per alcune sovrapposizioni con i passi 'corciresi' della versione di Giuseppe Zuccante $(1909)^{26}$ - e si segnalava per l'assenza di fraintendimenti della lettera, a dimostrazione che chi mise mano all'articolo doveva avere una buona padronanza della lingua greca ed essere versato nelle asprezze della prosa tucididea. Rilevare la mancanza di specifici errori grammaticali non significa però ammettere che le versioni raccolte nel centone fossero un esempio di resa fedele. $\mathrm{Al}$ contrario, esse mostravano a più livelli una deliberata manipolazione del testo di partenza.

Spicca, a questo riguardo, la frequente obliterazione di importanti dettagli legati alle tecnicalità del sistema ateniese, che, se richiamate, avrebbero contribuito ad allentare il nesso analogico tra passato e presente. Così, nel primo excerptum («La Marcia su Roma e i salvatori della Patria»), il centonatore decise di espungere il rimando al sistema dei misthoi e alla restrizione del corpo civico a 5000 unità ${ }^{27}$.

26 Giuseppe Zuccante, storico della filosofia e professore presso la Accademia scientificoletteraria di Milano, pubblicò la sua traduzione di Th. III, 82-83 all'interno di una fortunatissima monografia su Socrate: fonti, ambiente, vita, dottrina (Torino, 1909), vincitrice, nel 1911, del Premio Reale dell'Accademia Nazionale dei Lincei di Filosofia. La versione, che rielaborava quella storica di Francesco Boni (Firenze, 1835), godette di una discreta fortuna nella pubblicistica di settore (cf. e.g. A. BECCARI, "Le dottrine politiche dei sofisti e le istituzioni nella Grecia del V secolon, Convivium 2 [1930], p. 152) ed ebbe sicura circolazione all'interno del circolo gobettiano: Piero possedeva una copia personale del Socrate (oggi presso il Centro Studi Piero Gobetti di Torino), mentre Augusto Monti ne raccomandava l'adozione e la lettura nel «Saggio di catalogo d'una biblioteca scolastica per l'insegnamento del latino e greco in un Liceo-Ginnasio italiano» (A. Montı, Scuola classica e vita moderna, Torino, 1923, p. 204 e infra, p. 71 ss). Non è possibile dare conto in questa sede delle singole coincidenze formali tra il centone tucidideo e la traduzione di Zuccante: basti ricordare che queste si addensano soprattutto in tre excerpta (“Il ramoscello d'ulivo», "I "Ras" e l'amministrazione», “Discordie tra i "Ras"»), senza mai pregiudicare la sostanziale autonomia del centonatore nei confronti dell'originale greco.

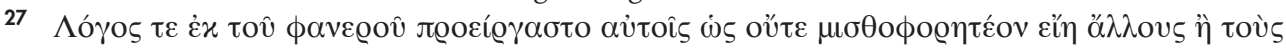

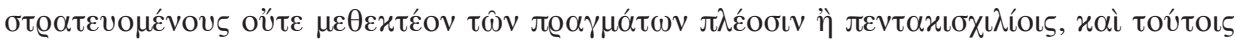

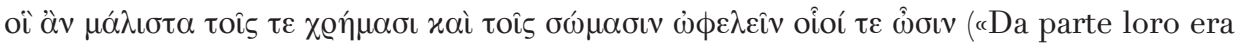
stata apertamente sparsa la voce che non si dovesse dare il soldo ad altri se non a chi faceva la guerra, e che non avrebbero dovuto partecipare alla vita politica più di cinquemila, e che dovevano essere quelli che potevano offrire maggiore utilità col loro denaro e le loro persone» [Th. VIII, 65, 3]) > «Da più tempo gli oligarchi avevano fatto 
Altre volte erano i titoletti a proporre un'interpretazione tendenziosa del greco: negli ultimi due brani, ad esempio, le didascalie (“I "Ras” e l'amministrazione»; “Discordie tra i "Ras")) attribuivano ai fedelissimi di Mussolini - sempre identificati con i gruppi oligarchici - una serie di comportamenti delittuosi che Tucidide riferiva anche alla fazione democratica. Un'ulteriore categoria di infedeltà - più legata al piano stilistico-formale - si manifestava infine nella sistematica eliminazione di parole, sintagmi e giri di frase considerati pleonastici o ripetitivi. Tali omissioni, che punteggiavano il dettato della versione italiana, erano motivate dal chiaro intento di promuovere una resa più incisiva e conforme ai canoni del linguaggio giornalistico ${ }^{28}$.

Il tratto più rilevante del modus vertendi del centonatore si esprimeva tuttavia nella marcata enfatizzazione della lettera, secondo una strategia amplificatoria che aumentava la pregnanza e la vis polemica del "corsivo", soprattutto nei luoghi in cui esso non considerava le rese di Zuccante. Tra i molteplici esempi che potremmo avanzare, segnaliamo una nutrita serie di espansioni, aggiunte e disambiguamenti che da un lato estremizzavano i sentimenti dei protagonisti (la temerarietà degli oligarchici, la compiacenza dello stato, la remissività del popolo), e, dall'altro, accentuavano le atrocità degli eversori:

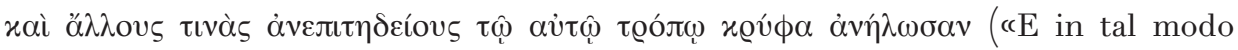
tolsero di mezzo di nascosto alcuni tra i nemici» [Th. VIII, 65, 2]) > «Quindi tutta la parte popolare fu presa d'assalto con un crescendo di uccisioni sistematiche» [“Gli assassini»]

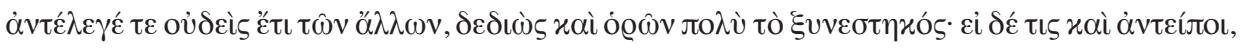

circolare la voce che tutti i diritti spettavano unicamente agli uomini di guerra ed ai soli cittadini capaci di servire la città con la persona e con gli averi» [“La Marcia su Roma e i salvatori della Patrias]. Qui e di seguito, il testo greco riproduce l'edizione di Giovan Battista Alberti (Thucydidis Historiae, ed. G.B. Alberti, I-III, Romae, 1972-2000), mentre le traduzioni citate - fatta eccezione per quelle del centone - sono di Franco Ferrari (cf. Tucidide, La Guerra del Peloponneso, introduzione di M.I Finley, traduzione a cura di F. Ferrari, bibliografia e note di G. Daverio Rocchi, Milano, 2016 ${ }^{14}$, ad locc.).

28 A queste esigenze vanno ricondotte le ricorrenti cassature di avverbi, attributi, perifrasi, che, nell'ottica dell'articolista, aggiungevano poco o nulla a frasi giudicate già di per sé troppo dense o estese, e che per questo apparivano bisognose di rimaneggiamenti e semplificazioni. A puro titolo esemplificativo, si considerino, nei soli primi due

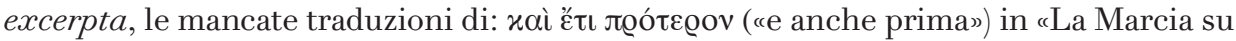

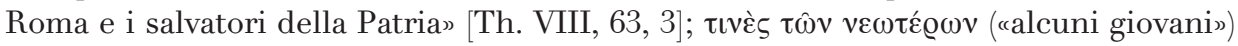
e x@ú $\phi \alpha$ (“di nascosto») in "Gli assassini» [Th. VIII, 65, 2]. Degna di segnalazione, per

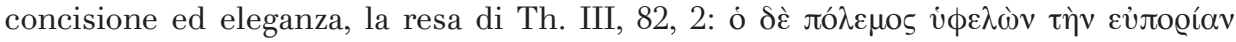

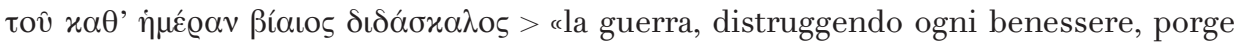
continue lezioni di violenza» (“L'insegnamento della guerra»). 


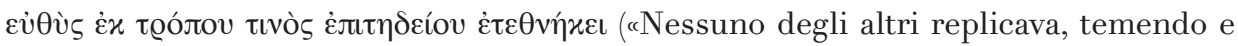
vedendo il gran numero dei congiurati, e se uno si opponeva, subito moriva in un modo adatto" [Th. VIII, 66, 2]) > “Così numerosa ed insolente era divenuta la fazione oligarchica, che non si trovava alcuno che osasse alzare la voce contro di essa. Se, per caso, qualche temerario l'avesse tentato, le più sanguinose vendette erano pronte a colpirlo» [“La paura»]

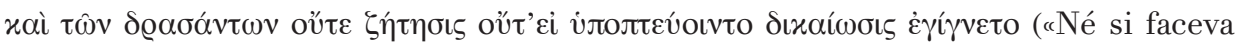
ricerca dei colpevoli né processo dei sospettati» [Th. VIII, 66, 2]) > «Lo Stato non si curava di ricercare i colpevoli di tanti misfatti. Piena impunità era accordata agli assassini, che, anche se conosciuti, potevano circolare liberamente» [“L'impunità degli assassini»]

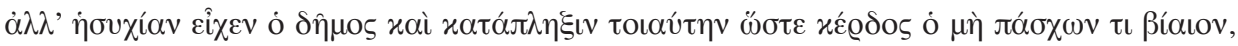

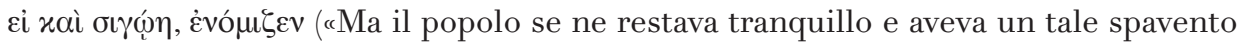
da considerare un guadagno se uno non subiva violenza, anche se taceva» [Th. VIII, $66,2])>$ «Il popolo non osava protestare. Egli vivea in tale stato di spavento, che si riputava già felice di poter sfuggire con il silenzio allo sterminio che lo minacciava» [«Il silenzio»]

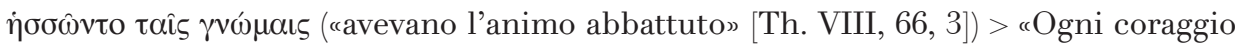
era prostrato. Un'aura di terrore travolgeva ogni cosa» [“I traditori»]

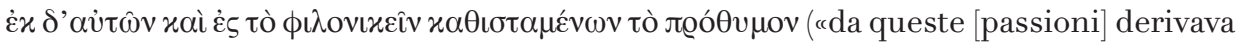
anche l'ardore di uomini posti di fronte alla necessità di vincere ad ogni costo» [Th. III, 82, 8]) > «Queste passioni travolgevano gli spiriti e li eccitavano a osare qualunque scelleratezza» [«Inflessibilmente»].

Così confezionata, l'antologia lasciava dunque trasparire un'articolata rielaborazione della fonte che coinvolgeva tanto il piano della dispositio quanto quello dell'elocutio. I brani prelevati dalle Storie non solo venivano redistribuiti in una sequenza originale, ma anche riformulati attraverso una serie di rese libere che potenziavano - e in parte alteravano - il dettato tucidideo. Quest'ultimo, sfrondato dalle informazioni inessenziali, risultava amplificato nell'intento di veicolare, con maggior incisività, precisi messaggi sugli avvenimenti contemporanei. L'esito, come anticipato, era quello di realizzare un'energica requisitoria contro l'autorità mussoliniana, spalleggiando le iniziative degli aventiniani. In quest'ottica, la parola dei classici si faceva anticipatrice - e in certo modo garante - di un vasto "programma di opposizione» che trovò piena formulazione in un editoriale gobettiano del 10 dicembre, in cui l'invocazione al «rovesciamento dell'oligarchia che tiene il governo" conservava l'eco delle suggestioni che avevano ispirato la composizione di «Tucidide e il fascismo»:

Le opposizioni devono superare il punto morto dell'attuale vita italiana dichiarandosi pronte alla successione. [...] Un governo così composto [...] garantirà i seguenti vantaggi: 1) sarà un governo di partiti responsabili e non di avventurieri e di dittatori; 2) 
sarà il primo governo che [...] parlerà col prestigio della democrazia, del consenso e di una parte delle classi proletarie; 3) invece che da un blocco di interessi personali, nascerà da una collaborazione leale e aperta di forze e di programmi diversi, ma non contradditori, controllati dagli istituti democratici moderni; 4) invece di essere uno Stato balcanico o sud-americano l'Italia si metterà sulla via di diventare uno Stato europeo moderno. [...] Se la forza dell'opinione pubblica favorevole a questo programma non sarà decisiva e concorde da rovesciare l'oligarchia che tiene il governo la battaglia avrà almeno guadagnato in chiarezza; la successione, più lontana, sarà più seria $^{29}$.

\section{"Classici della libertà»: Tucidide, Gobetti e l'interpretazione liberale della tradizione.}

La stretta interrelazione tra il riuso delle Storie e l'agenda politica di Gobetti apre la strada a un ulteriore approfondimento sulle premesse teoriche che animarono il centone tucidideo. La naturalezza con cui la fonte antica venne integrata nell'orizzonte ideologico-programmatico del liberalismo gobettiano spinge infatti a domandarsi se, nelle intenzioni di Piero, non esistesse un dialogo più profondo tra la parola di Tucidide e le istanze che sostanziarono l'esperienza politica de La Rivoluzione Liberale; se cioè, dietro alla provocatoria riappropriazione del passato proposta da "Tucidide e il fascismo", non esistesse un progetto di recupero della tradizione più ambizioso di quello dettato dalla pur impellente necessità di aggirare le maglie di una censura fattasi ormai opprimente. Tali interrogativi, che certo non vogliono sottostimare il peso assunto dai condizionamenti esterni imposti dal regime, paiono ulteriormente giustificati dalla costante propensione di Gobetti a favorire, in qualità di pubblicista ed editore, un ampio e appassionato ripensamento di opere e autori da lui giudicati fedeli alla causa della libertà; una libertà - è bene chiarirlo - che Piero concepiva, secondo l'efficace formulazione di Gioele Solari, sia “come espressione della personalità individuale», sia, meno crocianamente, come "attività di personalità collettive» impegnate nell'agone politico $^{30}$.

Espressione precoce di tale inclinazione fu la tesi di laurea di Gobetti, dedicata alla Filosofia politica di Vittorio Alfieri e discussa presso l'Università di Torino nel luglio 1922 sotto la guida dello stesso Solari. Il saggio, stampato l'anno successivo per la Piero Gobetti Editore, approfondiva l'attitudine libertaria e antidogmatica del pensiero alfieriano, individuando - nelle tragedie come negli scritti teorici -

29 P. Gobetti, “La successione», p. 185; corsivo mio.

30 Cf. A. Mautino, La formazione della filosofia politica di Benedetto Croce. Con uno studio sull'autore e la tradizione culturale torinese da Gobetti alla Resistenza di G. Solari, a cura di N. Воввіо, Bari, 1953, p. 64. 
una prima elaborazione di quei concetti di popolo e nazione che avrebbero offerto il lievito ideologico ai moti risorgimentali. Nel corso della sua indagine, però, Gobetti non si limitava a celebrare la «religione della libertà» dell'Alfieri ${ }^{31}$, ma finiva per cercare nell'astigiano il proprio pensiero, offrendo cpiù che una lettura fedele dello scrittore [...], una proposta politica nuova; per l'appunto il progetto della rivoluzione liberale ${ }^{32}$ ». Un atteggiamento fortemente attualizzante, dunque, che non rimase appannaggio del solo Piero, ma coinvolse l'intera rete dei suoi collaboratori, influenzando, tra gli altri, Augusto Monti, che proprio sotto la spinta della tesi gobettiana volle sperimentare un nuovo indirizzo pedagogico improntato alla lettura dei «nostri classici come 'classici della libertà'33»).

Se riesaminiamo allora in quest'ottica l'intera sequenza degli articoli di argomento storico pubblicati su La Rivoluzione Liberale nel '24, ci accorgiamo che la rivista, oltre ad agire sul piano della polemica politica, ambiva a delineare una raccolta - ma potremmo anche dire un canone - di voci autenticamente liberali o comunque integrabili nell'alveo del liberalismo gobettiano. Tali erano senz'altro quelle di due anti-fascisti intransigenti come Francesco Saverio Nitti e Guglielmo Ferrero, autori, rispettivamente, del saggio sui moti napoletani del 1820 e dei passi 'anti-cesariani' antologizzati in “La dittatura di Cesare». Ad essi si potevano affiancare, quasi naturalmente, i nomi di Massimo D’Azeglio e Luigi Carlo Farini, patrioti e membri di spicco del gruppo dirigente sabaudo che avviò, insieme a Cavour, un processo di modernizzazione dell'Italia presentato dallo stesso Piero nei termini di una «rivoluzione liberale» ante litteram $^{34}$. Infine, Machiavelli e Sallustio: il primo, più volte celebrato da Gobetti come precursore del libertarismo dell'Alfieri, aveva avuto il merito di instaurare una «religiosità civile» fondata sulla (spontaneità di iniziative e di economia ${ }^{35}$ ); il secondo, severo critico dell'eversore Catilina, fu anch'esso autore alfieriano d'elezione, non solo perché letto e tradotto dall'astigiano per più di un ventennio, ma anche per la sua tempra anti-tirannica, riconosciutagli nell'esergo del trattato-manifesto Della Tirannide (1789): impune quaelibet facere, id est regem esse (Sall. Iug. 31.26) ${ }^{36}$.

31 P. Goвetti, La filosofia politica di Vittorio Alfieri, Torino, 1923, p. 97.

32 A. Fabrizi, “Postfazione», in P. Goвetтi, La filosofia politica di Vittorio Alfieri, Roma, 2012, p. 146.

33 A. Monti, “Con Piero Gobetti vivo e morto», Belfagor 11 (1956), p. 205. Sul rapporto tra Monti e Gobetti, vd. infra p. 71ss.

34 P. Gobetti, La Rivoluzione Liberale. Saggio sulla lotta politica in Italia, a cura di E. Alessandrone Perona, Torino 2008, p. 23.

35 Ivi, p. 12. Per una recente sintesi dell'interpretazione gobettiana del pensiero di Machiavelli, vd. P. Bagnoli, “Piero Gobetti e Machiavelli», in L.M. Bassani-C. Vivanti (a cura di), Machiavelli nella storiografia e nel pensiero politico del XX secolo, Milano, 2006, p. 93-100 e L. Mitarotondo, Un 'Preludio', p. 55-82.

36 Sul contributo di Sallustio alla definizione del pensiero antitirannico di Alfieri 
Non diversamente, spostando lo sguardo all'insieme delle uscite nel quadriennio '22-'25, riscontriamo che i classici presentati al pubblico della rivista - in forma di citazione o antologia - erano sempre organici agli indirizzi ideologici del gruppo gobettiano. Così, all'amato Machiavelli, già centonato nel corso del '23, si aggiungevano un lungo brano del Pamphlet des Pamphlets di Paul Louis Courier - vero e proprio inno alla libertà di stampa nella Parigi restaurata -, stralci da John Stuart Mill e una pagina delle Ricerche sopra la natura e le cause della ricchezza delle Nazioni del liberista Adam Smith ${ }^{37}$. Allo stesso modo, compulsando il catalogo della Piero Gobetti Editore, osserviamo che l'unico classico ripubblicato tra '23 e '25 fu il trattato On liberty del Mill (1859), la cui riedizione - in traduzione italiana prese le mosse nel corso dell'autunno del '23 per concludersi, con qualche ritardo, agli inizi del '25 ${ }^{38}$. L'uscita, del cui valore politico era difficile dubitare, veniva così salutata da Luigi Einaudi:

In tempi di mortificazione dello spirito, quando, per fiaccare le voci dei ribelli, si assevera dai dominatori la unanimità del consenso interno, necessaria affinché la patria vigoreggi e sia rispettata dallo straniero, giova rileggere i grandi libri sulla libertà. [...] A queste proposizioni mortificatrici [...] il saggio del Mill oppone la giustificazione logica del diritto al dissenso e la dimostrazione della utilità sociale e spirituale della lotta ${ }^{39}$.

Alla luce di questo quadro, viene insomma spontaneo domandarsi quali fossero i presupposti sulla base dei quali era possibile integrare anche Tucidide tra i “classici della libertà» gobettiani, sorvolando sulla sua ben nota predilezione

cf. S. CAsini, “'L'inestricabile labirinto'. Alfieri traduttore e le versioni da Sallustio», in A. Bruni-R. Turchi (a cura di), A gara con l'autore. Aspetti della traduzione nel Settecento, Roma, 2004, p. 253ss., con P. Pellizzari, “Alfieri sulle orme di Sallustio (qualche spunto di ricerca)", Seicento e Settecento 5 (2010), p. 154ss. L'esergo sallustiano, assente nella prima stesura dell'opera (1777), venne inserito nell'editio princeps di Kehl (1789-90; cf. $i v i$, p. 155-156). Le istanze politico-ideologiche che furono alla base dell'interesse alfieriano per Sallustio furono oggetto di dibattito all'interno del circolo di Gobetti; vd. le osservazioni di Umberto Calosso, risalenti proprio al 1924: «la Catilinaria tradotta è, non solo cronologicamente, la prima operetta politica dell'Alfieri, quasi una premessa alle altre che prenderanno questo nome»(U. Calosso, L'anarchia di Vittorio Alfieri. Discorso critico sulla tragedia alfieriana, Bari, $1949^{2}$, p. 40).

37 N. Machiavelli, “Consigli machiavellici ai nepoti», p. 127; J.S. MiLl, “La libertà», $R L$ II (1923), n. 36, p. 148; N. Machiavelli, "Commento a un preludio", p. 77; P.L. Courier, “Pamphlet des Pamphlets», RL III (1924), n. 29, p. 117; A. Sмiтн, “L'opinione di Smith», RL IV (1925), n. 40, p. 161.

38 Cf. N. Urbinati, “Postfazione», in J.S. Mill, La libertà, Roma, 2011, p. 186 e A. Pedio, "Scheda», ivi, p. 195-198.

39 L. Einaudi, «Prefazione», in G.S. Mill, La libertà, Torino, 1925, p. I, IV. 
per il 'principato' di Pericle (Th. II, 65, 9) e sulle severe critiche da lui rivolte alla democrazia ateniese ${ }^{40}$. Al netto di tali ostacoli - dei quali è peraltro difficile stabilire fino a che punto la cerchia di Gobetti fosse consapevole - una prima, diretta motivazione poteva senz'altro risiedere nel giudizio che Tucidide pareva esprimere, attraverso la sua narrazione, sul putsch oligarchico del 411 e sugli esiti eversivi della stasis corcirese. Il tono del resoconto, severo e accorato, doveva infatti apparire a Piero e ai suoi collaboratori come una netta condanna di quei metodi sovversivi che avevano ispirato anche la "rivoluzione" fascista, significativamente definita dallo stesso Gobetti come «il colpo di Stato compiuto da un'oligarchia mediante l'umiliazione di ogni serietà e coscienza politica ${ }^{4{ }^{1} \text { ). }}$. Com'era avvenuto nel caso di Sallustio - che nella figura di Catilina rappresentava un archetipo negativo di politico-capobanda riferibile a Mussolini ${ }^{42}$ - le pagine tucididee sanzionavano una concezione violenta e illiberale della lotta politica che contraddistingueva anche l'esperienza fascista, sollecitando una naturale e quasi spontanea approvazione da parte di chi, come l'editore torinese, era quotidianamente impegnato a denunciare i frutti avvelenati del sovversivismo mussoliniano.

Ciononostante, queste non appaiono le uniche basi teoriche sulle quali è possibile concepire un accostamento tra Tucidide e l'universo ideologico de $\mathrm{La}$ Rivoluzione Liberale. Altre importanti indicazioni provengono dai giudizi formulati

$40 \mathrm{Si}$ ricordi, a puro titolo esemplificativo, che nella Germania post-Versailles l'ispirazione autocratica del governo pericleo adombrata in Th. II, 65, 9 fu spesso enfatizzata per stigmatizzare - e contrario - i limiti della democrazia weimariana, cf. P. Butti de Lima, “Tucidide fra i pensatori della politica», Anabases 8 (2008), p. 260ss.; A. Andurand, “Lectures allemandes de l'Oraison Funèbre de Périclès (1850-1930)", in V. Fromentin-S. Gotteland-P. Payen (éd. par), Ombres de Thucydide. La réception de l'historien depuis l'Antiquité jusqu'au début du XXe siècle, Bordeaux, 2010, p. 578ss., V. Azoulay, Pericle. La democrazia ateniese alla prova di un grand'uomo, Torino, 2017 [trad. it. di Périclès. La démocratie athénienne à l'épreuve du grand homme, Paris, 2016²], p. 208-213.

41 P. Goвetтi, “La tirannide», $R L$ I (1922), n. 33, p. 123. Corsivo mio.

42 Cf. la descrizione di Mussolini abbozzata nel maggio '22: “Io non riesco ad immaginarmi Mussolini altrimenti che sotto le spoglie del più audace e torbido condottiero di compagnie di ventura; o talora meglio come il capo primitivo di una selvaggia banda posseduta da un dogmatico terrore che non consente riflessioni» (P. Goвetтi, “Uomini e idee», RL I [1922], n. 15, p. 56). Per uno sguardo più ampio sulla complessa - e per certi versi contradditoria - ricezione di Catilina nella cultura italiana post-unitaria e fascista vd. N. CRiniti, “La tradizione catilinaria: interpretazioni provinciali italiane tra le due guerre mondiali», Aevum 42 (1968), p. 114-120; ID., "Interpretazioni storiche catilinarie nell'Italia unita», Nuova Rivista Storica 52 (1968), p. 355-400; ID., "Schegge sulla fortuna di Catilina nell'Italia del XX secolon, in Philias Charin. Miscellanea di studi classici in onore di Eugenio Manni, II, Roma, 1979, p. 611-627. 
sull'opera dello storico da alcune voci di sicura rilevanza nella formazione intellettuale e civile di Gobetti. Tra queste spicca quella di Gaetano De Sanctis, docente di storia antica presso l'Università di Torino dal 1900 al 1929 e dotato secondo la testimonianza dello stesso Piero - di «una certa intonazione di vero maestro ${ }^{43}$ ». “Amico entusiasta» delle iniziative gobettiane fin dal ' $18^{44}$, De Sanctis si confermò, nonostante il fervente cattolicesimo, un punto di riferimento ideale per il periodico, ottenendo sul numero 7 de La Rivoluzione Liberale (2 aprile '22) un ampio articolo - firmato da Natalino Sapegno - che tracciava un bilancio elogiativo della sua produzione storiografica ${ }^{45}$. Tale riconoscimento, riservato dalla rivista a pochissimi contemporanei, si spiega col fatto che De Sanctis, per la generazione di Gobetti, non rappresentava soltanto uno degli studiosi più autorevoli nel panorama nazionale, ma anche un modello di antifascista intransigente e rispettato ${ }^{46}$. La sua stessa attività di storico, pur maturata alla scuola positivista del Beloch, aveva saputo abbinare al rigore metodologico una convinta apertura nei confronti del pensiero storiografico crociano e in particolare a quella concezione della storia come "storia della libertà» condensata dalla celeberrima dedica del IV volume della Storia dei Romani, vergata proprio a ridosso dell'ottobre '22: “a quei pochissimi che hanno parimente a sdegno d'essere oppressi e di farsi oppressori ${ }^{47}$ ».

43 “In questa scellerata università di Torino [...] (in Lettere e filosofia) ci sono due persone intelligenti: De Sanctis di storia antica, [...] serio, aristocratico (in senso buono) e con una certa intonazione di vero maestro, e il simpaticissimo Farinelli, buono, entusiasta, poeta, anima fervida di maestro» (lettera di Gobetti a Santino Caramella, 8 gennaio 1919; cf. P. Gobetti, Carteggio 1918-1922, a cura di E. Alessandrone Perona, Torino, 2003, p. 20). Alla luce di questa testimonianza pare lecito accostare il De Sanctis a quei “maestri provenienti dall'alta cultura prevalentemente universitaria [Croce, Einaudi, Salvemini, Gentile]», che Norberto Bobbio annoverava tra le «fonti dirette» del pensiero gobettiano, cf. N. Воввіо, Italia fedele. Il mondo di Gobetti, Firenze, 1986, p. 51.

44 P. Gobetri, Carteggio, p. 20. L'epistolario gobettiano del '18-'19 testimonia alcuni contatti diretti con il De Sanctis; nelle parole di Piero, lo studioso romano manifestava “approvazione e incoraggiamento» per le prime attività pubblicistiche del giovane Gobetti (cf. ivi, p. 8, 20, 23-24, 41-42). È significativo che tra le carte desanctisiane sia stato rinvenuto l'annuncio del primo numero de La Rivoluzione Liberale, cf. M. Accame LANZILlotTa, Le edizioni, p. 11.

45 L'articolo «Gaetano De Sanctis» (RL I [1922], n. 7, p. 27) inaugurava una rubrica dedicata ai protagonisti della ricerca storica italiana; il secondo medaglione fu dedicato - sempre da Sapegno - a Gaetano Salvemini (RL I [1922], n. 25, p. 93).

46 Sulla militanza desanctisiana nelle file del PPI e in alcune associazioni cattoliche piemontesi, vd. S. Accame, Gaetano De Sanctis fra cultura e politica. Esperienze di militanti cattolici a Torino (1919-1929), Firenze, 1975, е A. Aмico, Gaetano De Sanctis. Profilo biografico e attività parlamentare, Tivoli, 2007, p. 70-102.

47 Sul tema della libertà politica nella storiografia desanctisiana di argomento greco 
Quanto a Tucidide, giova ricordare che lo storico ateniese esercitò sempre una profonda influenza sul modo in cui De Sanctis guardò alle vicende greche: «se c'è un punto - ha giustamente notato Massimiliano Pavan - che il De Sanctis [...] recepisce senza riserve [da Tucidide] è la concezione non solo ellenocentrica, ma atenocentrica della storia greca, in linea di primato morale e civile prima che politico ${ }^{48}$ ). Tale primato si identificava per De Sanctis con gli ideali di giustizia sociale e di tutela delle libertà individuali che la democrazia periclea aveva saputo porre alla base del proprio ordinamento e che aveva avuto il compito di diffondere nel Mediterraneo attraverso la propria egemonia politico-militare. Questa vocazione civilizzatrice, poi tradita dallo sviluppo predatorio dell'imperialismo attico, conservava per lo studioso un valore universale e trovava la sua più alta formulazione proprio nell'epitaffio di Pericle tramandatoci da Tucidide ${ }^{49}$. L'orazione, per De Sanctis, coincideva addirittura con le ultime pagine scritte dallo storico e ne costituiva un vero e proprio testamento spirituale, oltreché, naturalmente, un lascito perenne che ccommuove sempre tutti gli amici della libertà ${ }^{50}$ ).

vd. la sintesi di D. Piovan, “Il fascismo e la storia greca», in J. BAssi-G. Canì (a cura di), Sulle spalle degli antichi. Eredità classica e costruzione delle identità nazionali nel Novecento, Milano, 2014, p. 27-31; per i complessi rapporti con l'opera di Croce, non immuni da dissensi metodologici, cf. A. Momigliano, "In memoria di Gaetano De Sanctis (1870-1957)", Rivista Storica Italiana 69 (1957), ora in ID., Secondo contributo alla storia degli studi classici e del mondo antico, Roma, 1960, p. 313-316; G. SAsso, Il guardiano della storiografia. Profilo di Federico Chabod e altri saggi, Napoli, 1985, p. 193-202; E. Gавва, Cultura classica e storiografia moderna, Bologna, 1995, p. 246, 250-256; F. Santangelo, “Uno 'storico di battaglie': lo stile di Gaetano De Sanctis», in A. Giavatto-F. Santangelo (a cura di), La Retorica e la Scienza dell'Antico. Lo stile dei classicisti italiani nel Ventesimo secolo, Heidelberg, 2013, p. 34-70, passim. Infine, sulla cronologia esatta di Storia dei Romani, IV.1, cf. L. Polveriñ “Vita magistra historiae'. La concezione storica di Gaetano De Sanctis nella Storia dei Romani», in S. CAgnazzi et al. (a cura di), Scritti di storia per Mario Pani, Bari, 2011, p. 400.

48 M. PAvan, "Gaetano De Sanctis e la democrazia periclea», Clio 19 (1983), p. 25.

49 Per l'articolato giudizio desanctisiano sulla democrazia periclea (in parte senz'altro negativo), vd. ivi, p. 17-29; su Tucidide “critico dell'impero» nell' interpretazione di De Sanctis, vd. il recentissimo D. Piovan, Tucidide in Europa. Storici e storiografia nell'età dello storicismo, Milano, 2018, p. 49-75. Un agile ed efficace profilo del De Sanctis storico greco si trova in A. Momigliano, In memoria, p. 304-308, 314-315 e C. Ampolo, Storie greche. La formazione della moderna storiografia sugli antichi Greci, Torino, 1997, p. 100-103.

50 G. De Sanctis, Problemi di storia antica, Bari, 1932, p. 185. Notevole la successiva canonizzazione dell'epitaffio tra i cprecursori ideali del Mazzini. Il quale era nella loro linea, sebbene con consapevolezza infinitamente maggiore, quando il suo ideale 
Tali idee - espresse nella forma più compiuta nel dittico monografico della vecchiaia: Storia dei Greci (vol. II; 1939) e Pericle (1944) - ebbero significative anticipazioni già negli scritti e nei seminari del period o torinese; gli stessi che Piero e alcuni suoi collaboratori lessero e frequentarono. Così, la parabola dell'Atene democratica veniva tracciata - con importanti parallelismi rispetto ai saggi degli anni '30 e '40 - nei capitoli finali della seconda edizione di Atthis. Storia della Repubblica Ateniese dalle origini alla età di Pericle (Torino, 1912), testo annotato da Gobetti nell'aprile $1920^{51}$ (fig. 2) e studiato da Sapegno nell'estate del '21 in vista dell'esame di storia antica, per il quale egli aveva compulsato metodicamente tutti i libri e gli appunti del maestro ${ }^{52}$. E proprio da una serie di dispense del 1921/2 apprendiamo che in quell'anno accademico De Sanctis dedicò un intero corso alla storia greca di V secolo, “con particolare riguardo agli avvenimenti accaduti tra le guerre persiane e quelle del Peloponneso", illustrati a partire da una nutrita selezione di passi tradotti e commentati dalla pentekontaetia (Th. I, 89-118) ${ }^{53}$. Tra i temi prediletti c'era dunque, di nuovo, l'imperialismo ateniese, ma uno spazio rilevante veniva dedicato anche all'intricata vicenda compositiva delle Storie, in merito alla quale De Sanctis sviluppava posizioni in parte affini a quelle che avrebbe poi sostenuto nei contributi successivi e che riconoscevano nel logos epitaphios il testamento morale di Tucidide ${ }^{54}$. Se Sapegno non fu verosimilmente tra gli studenti di quel corso, un altro sodale di Gobetti, Mario Attilio Levi, dovette

dell'Italia libera [...] s'illuminava e acquistava valore universale nell'ideale della Europa libera e della libera umanità» (ibid.).

51 Il volume fa parte della biblioteca personale di Piero e reca sul frontespizio la seguente nota di possesso autografa: “Piero Gobetti. 28 aprile 1920». Sottolineature attribuibili a Gobetti si concentrano soprattutto nelle prime sezioni del testo. Ringrazio la dott.ssa Franca Ranghino per l'assistenza nella consultazione autoptica della stampa presso il Centro Studi Piero Gobetti di Torino.

52 «Studio anche gli appunti di storia antica, e fra poco incomincerò anche a leggere i libri di De Sanctis: guarderò, se mi sarà possibile, di far quel lavoro che mi avevi fatto promettere per la rivista [sc. l'articolo poi pubblicato su $R L$ nell'aprile '22, dove si discute anche l'Atthis]) (lettera di Sapegno a Gobetti, 13 settembre 1921; P. Goвetti, Carteggio, p. 229).

53 Di queste dispense ha dato notizia - riassumendone i contenuti e citandone significativi estratti - Eugenio Lanzillotta (cf. E. Lanzillotta-V. Costa, “La riscoperta di Tucidide presso la Scuola Romana tra Ottocento e Novecento", in Ombres de Thucydide, p. 563 ss.).

54 Ivi, p. 558-562, 568-569. Le conclusioni presentate nelle dispense sviluppavano a loro volta osservazioni già contenute in un altro ciclo di lezioni risalente ai primi anni torinesi - a.a. 1901/2 (ivi, p. 563-568). Significativo che una copia di queste prime dispense sia oggi conservata presso il Centro Gobetti come parte del lascito di Franco Antonicelli, anch'egli in rapporti di amicizia con Piero (cf. P. Goвeтті, Carteggio, p. 243). 


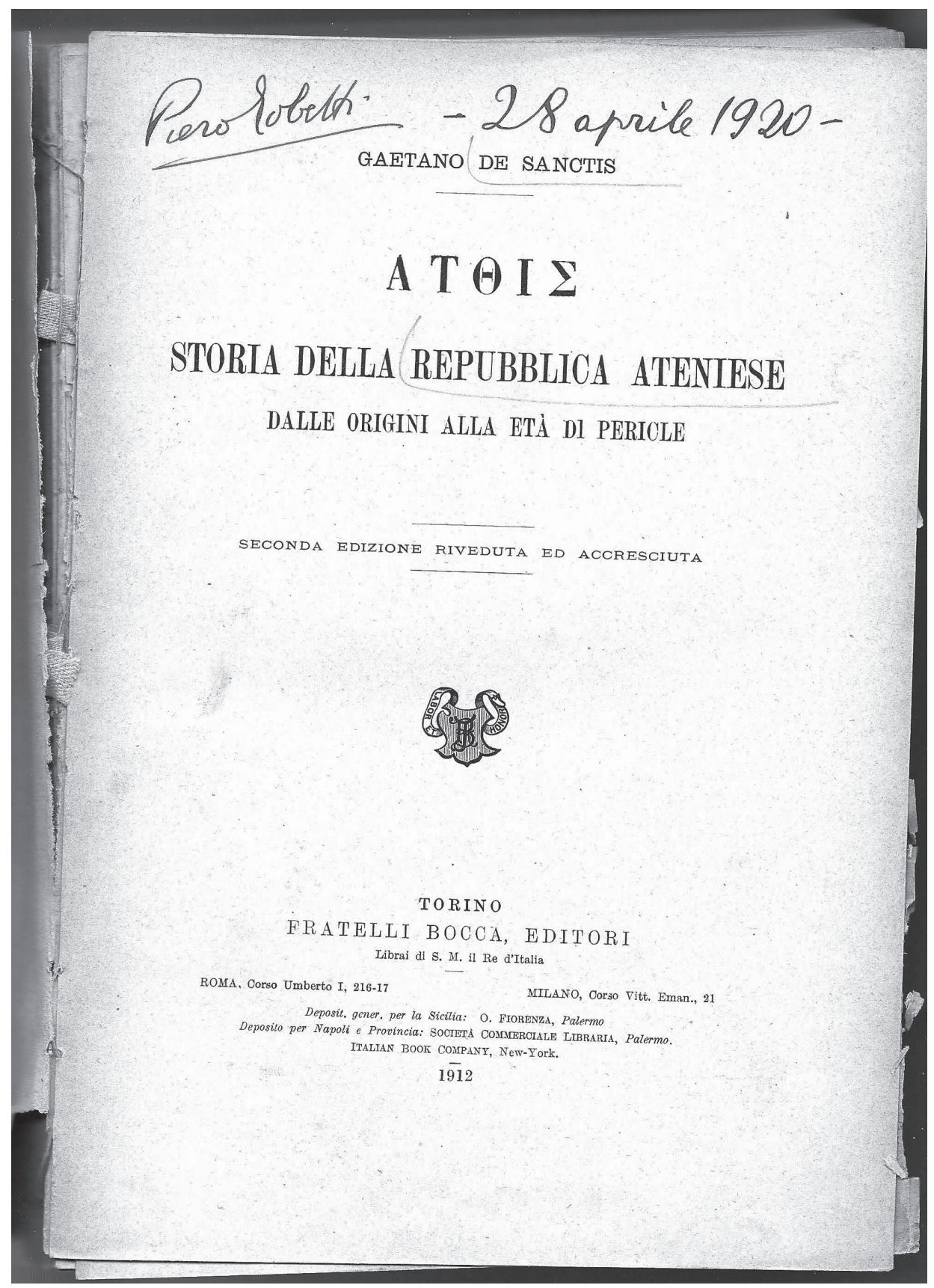

Fig. 2 Frontespizio di G. De Sanctis, Atthis. Storia della Repubblica Ateniese dalle origini alla età di Pericle, Torino, $1912^{2}$, con nota di possesso autografa di Piero Gobetti. Torino, Centro Studi Piero Gobetti. 
frequentare almeno una parte delle lezioni: allievo diretto del De Sanctis, Levi era da tempo collaboratore de La Rivoluzione Liberale e, nonostante le precoci simpatie fasciste, si dimostrò fino al '22 un convinto sostenitore delle iniziative di Piero, animando, insieme ad un'altra brillante classicista di scuola desanctisiana, Elena Valla, i dibattiti che si tenevano quotidianamente presso il cenacolo di casa Gobetti ${ }^{55}$.

Se dunque appare verosimile ipotizzare che il magistero di De Sanctis sia stato decisivo nella formulazione di un'immagine di Tucidide organica al programma ideologico de LaRivoluzione Liberale, un'altra indiscussa auctoritas del firmamento gobettiano - Vittorio Alfieri - confortava tale giudizio. L'astigiano, come noto, non fu solo l'autore prediletto di Gobetti ma anche un modello di intellettuale con cui il giovane torinese volle sempre confrontarsi, alimentando un cpresupposto autobiografico» che - nelle parole di Mario Fubini - lo portò “a confondersi col suo autore, a continuarne il discorso, e integrarne, sino a trasfigurarlo talvolta, il pensiero ${ }^{56}$ ). Alla luce di tali premesse e di un più generale 'alfierismo' dell'intera cerchia gobettiana ${ }^{57}$, pare dunque rilevante richiamare la celebrazione di Tucidide

55 “A Torino ho una diecina di amici (Fubini, M. Marchesini, A. Marchesini, A. Prospero, E. Valla, M. A. Levi, N. Sapegno, G. Stolfi, e qualche altro meno attivo) che [...] vengono a casa mia, discorrono, discutono, pensano. Al lavoro di redazione non bada nessuno" (lettera di Gobetti a Caramella, 18 febbraio 1920; P. Goвeтті, Carteggio, p. 99). Sul rapporto tra Levi, Valla e Gobetti, cf. le note di Ersilia Alessandrone Perona ivi, p. 495-496, 520-521, e, sul discepolato di Levi presso il De Sanctis, la testimonianza autobiografica di M.A. Levi, "Alla scuola di De Sanctis negli Anni Venti», Storia della Storiografia 16 (1989), p. 5-14, con A. D'Orsi, "Storie di storici. Torino fascista", in P.G. Zunino (a cura di), Università e accademie negli anni del fascismo e del nazismo, Firenze, 2008, p. 395ss. Da ricordare che l'interesse desanctisiano per l'opera di Tucidide alimentò le ricerche di altri due brillantissimi allievi torinesi, Aldo Ferrabino e Arnaldo Momigliano, che nel corso degli anni Venti pubblicarono, rispettivamente, una monografia sull'Impero atheniese (1927) e un lungo articolo su La composizione della storia di Tucidide (1929); su questi temi vd. D. Piovan, "Un moderno interprete tucidideo: Aldo Ferrabino», Patavium 7 (1996), p. 63-94 ID., “Tucidide, Momigliano e lo storicismo", Quaderni di storia 45 (1997), p. 49-80 e ID. Tucidide in Europa p. 77-130, che ben illustrano le differenze tra gli approcci di Ferrabino e Momigliano al testo tucidideo e le loro prese di distanza da alcune posizioni del maestro.

M. Fubini, Ritratto dell'Alfieri e altri studi alfieriani, Firenze, 1967, p. 255-256 con N. Bоввіо, Italia fedele, p. 51.

57 Per limitarsi ad alcuni dei collaboratori più stretti di Gobetti, nel 1921 Umberto Calosso pubblicò quattro articoli di tema alfieriano su L'Ordine Nuovo e, nel 1924, l'importante monografia L'anarchia di Vittorio Alfieri (supra n. 36). Dal 1923, iniziarono a uscire i primi lavori alfieriani di Mario Fubini, mentre quelli di Natalino Sapegno datano agli anni Quaranta, ma già nell'agosto 1920 Sapegno dichiarava a Piero la propria passione 
contenuta in alcuni celebri capitoli del trattato Del Principe e delle Lettere (1789), opera largamente citata dallo stesso Piero nella tesi di laurea e da lui considerata essenziale per la ricostruzione delle coordinate teoriche del libertarismo dell'astigiano.

Come noto, Alfieri giudicava Tucidide nel contesto di una più ampia classificazione 'politica' degli scrittori, che tendeva a contrapporre due categorie ben distinte di letterati: da un lato, quelli “di principe», le cui opere curavano “assai più la eleganza del dire, che non la sublimità e forza del pensare»; dall'altro, i letterati vissuti in regime di libertà, ‘i quali [...] essendo più maschi, più veritieri, incalzanti, e feroci, $[. .$.$] non sono mai di ragione di principe { }^{58}$ ». L'appartenenza a quest'ultimo gruppo costituiva poi un'indispensabile premessa allo sviluppo dei "quattro ingredienti che componevano il sublime scrittore» (“alto animo, libere circostanze, forte sentire, ed acuto ingegno»), a loro volta decisivi per rendere ogni opera letteraria cun possente stimolo [...] al praticare, amare, e difendere la libertà ${ }^{59}$,. In quest'ottica, Tucidide, vissuto nella democratica Atene e non condizionato da nessun legame di dipendenza politica, andava senz'altro annoverato tra gli autori "figli di libertà e di virtù» e il suo nome poteva spiccare, in posizione rilevata, tra i ctanti altri scrittori del vero, che se tutti non nacquero liberi, indipendenti vissero almeno, e non protetti da nessuno»:

Tali [...] sono, per esempio, Demostene, Tucidide, Eschilo, Sofocle, Euripide, Cicerone, Lucrezio, Sallustio, Tacito, Giovenale, Dante, Machiavelli, Bayle, Montesquieu, Milton, Locke, Robertson, Hume ${ }^{60}$.

Questa classificazione, in certa misura meccanica e sbrigativa, trovava ulteriore conferma in altri passi del trattato, che - a partire dai medesimi presupposti - discutevano temi più circoscritti. Così, abbozzando un canone degli «storici sommi», l'attenzione di Alfieri si appuntava nuovamente sul «libero sprotetto» Tucidide, il cui “robusto conciso pensare e sentire» veniva magnificato come manifestazione del genio greco, “inventore d'ogni cosa perché [... libero ${ }^{61}$ ».

per l'astigiano (P. Goвetтi, Carteggio, p. 144). Sempre nel '23, un altro vorace lettore di Alfieri, Augusto Monti, ricavò da una lettera alfieriana al Caluso (28 marzo 1801) il

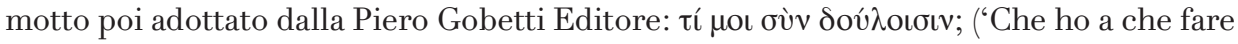
io con gli schiavi?'). Sull'‘alfierismo' della cerchia gobettiana, cf. spec. A. FABrizi, 'Che ho a che fare io con gli schiavi?'. Gobetti e Alfieri, Firenze, 2007 e ID., “Postfazione», in P. Goветті, La filosofia politica, p. 131-151.

58 Del Principe e delle lettere I, 3, cf. V. Alfieri, Della Tirannide, Del Principe e delle lettere, La virtù sconosciuta, a cura di M. Cerruti-E. Falcomer, Milano, 20115 , p. 204-205.

59 Del Principe e delle lettere III, 2 e 4 (ivi, p. 301, 317).

60 Del Principe e delle lettere I, 3 (ivi, p. 205-206).

${ }^{6}$ Del Principe e delle lettere II, 9 (ivi, p. 279-284). 
E sulla scia del medesimo topos, lo storico era di nuovo ricordato nella parte finale dell'opera tra i «sublimi» figli di Atene, «madre d'ogni sforzo di politica virtù e di un così bel vivere e libero e civile ${ }^{62}$ ». Senza volerci dilungare ulteriormente, è facile immaginare fino a che punto giudizi come questi - abbinati alle considerazioni di De Sanctis e al severo sguardo tucidideo sugli avvenimenti del 411 - potessero favorire la consacrazione dello storico tra i “classici della libertà», consentendone la valorizzazione all'interno di una strategia editoriale che proponeva un modello di riappropriazione del passato del tutto antipodale rispetto a quello anti-democratico coltivato dal fascismo.

\section{Classicismo e modernità: sotto il segno di Augusto Monti}

Le linee di frattura tra classicismo gobettiano e classicismo fascista non si riducono tuttavia alla sola, prevedibile divergenza sull'opzione liberal-democratica; esse ci appaiono più profonde e possono essere meglio comprese esaminando un'ultima, spinosa questione posta dal centone tucidideo: quella relativa alla sua incerta paternità. Se infatti dobbiamo immaginare che Gobetti sposò convintamente una lettura in senso liberale dell'opera di Tucidide, è altamente improbabile che sia stato lui in prima persona a confezionare l'antologia. Certo, Piero possedeva una buona conoscenza della lingua greca, ma dopo la licenza liceale dovette esercitarla solo sporadicamente e all'interno di prove traduttive assai circoscritte, essenzialmente riconducibili all'estate-autunno 192063; non possiamo poi ignorare che nell'imponente produzione gobettiana - circa duemilacinquecento pagine nei tre volumi della raccolta einaudiana - manca ogni riferimento allo storico ateniese ${ }^{64}$ e nessuna copia delle Storie risulta conservata nella pur vasta biblioteca dell'intellettuale torinese ${ }^{65}$. Alla luce di tali premesse, pare quindi inevitabile voler

62 Del Principe e delle lettere III, 4 (ivi, p. 317).

63 La corrispondenza gobettiana con Giovanni Papini e Santino Caramella del giugnonovembre 1920 documenta un progetto di traduzione delle vite e dei frammenti degli stoici tràditi da Diogene Laerzio (P. Gobetri, Carteggio, p. 126-128, 130-131, 174). L'opera, mai portata a termine, resta l'unica testimonianza a me nota di un impegno di Gobetti come traduttore di testi classici.

64 Cf. Opere di Piero Gobetti, a cura di P. Spriano et al., I-III, Torino, 1960-74. Tra le altre carte gobettiane edite, ho potuto reperire un unico, cursorio riferimento all'opera di Tucidide in un abbozzo di tesi risalente al '20-'21, intitolato La filosofia politica nel mondo classico, poi abbandonato a favore del progetto alfieriano. Nell'indice tematico dell'abbozzo compariva una sezione dedicata a "Gli storici: Erodoto, Tucidide, Senofonte», cf. P. Gobetтi, Carteggio, p. 452-453.

65 La biblioteca di Piero contava all'altezza del 1926 ca. 3120 titoli; la sezione greca ospitava molte opere di filosofia (tra cui Platone, Aristotele, i presocratici, Epicuro e 
individuare in altra persona, e in particolare nel profilo di uno stretto collaboratore di Gobetti, Augusto Monti, il probabile estensore di “Tucidide e il fascismo».

Titolare della cattedra di italiano e latino al liceo Massimo D’Azeglio di Torino, Monti non solo possedeva le competenze necessarie per produrre una traduzione originale e corretta degli excerpta tucididei; egli costituiva anche il punto di riferimento dell'intera cerchia gobettiana nel campo degli studi classici. Autore di oltre quaranta articoli per La Rivoluzione Liberale - tra cui quello affiancato, a mo' di pendant, al corsivo tucidideo ${ }^{66}$-, Monti era stato nominato proprio nel novembre '24 responsabile della sezione di “letterature classiche» del neonato Baretti $^{67}$, nel cui comitato redazionale egli esercitò un ruolo di primo piano fino al 1928, quando il periodico venne chiuso per intervento delle autorità fasciste. Alla sua iniziativa andavano inoltre ascritti, con ogni probabilità, tanto la provocatoria scelta dell'epigramma archilocheo che identificava la figura di Mussolini con quella del tiranno Leofilo (RL I [1922], n. 32), quanto il centone sallustiano pubblicato due anni dopo (RL III [1924], n. 38 $)^{68}$. Se insomma tutti gli indizi invitano ad assegnare ad Augusto Monti la paternità di “Tucidide e il fascismo», va comunque precisato che l'articolo, per quanto frutto delle competenze filologiche del singolo, esprimeva il punto di vista dell'intero gruppo gobettiano, del quale il classicista condivideva non solo l'interpretazione storica del quadro politico, ma anche il generale approccio alla tradizione letteraria ${ }^{69}$. Ciò considerato, è proprio partendo da questo rapporto di reciproca collaborazione tra Monti e Gobetti, che appare possibile definire con maggior precisione i presupposti ideologici che animarono il classicismo gobettiano, chiarendone al contempo le nette divergenze rispetto a quello «di regime» alimentato dalla propaganda fascista.

A questo riguardo, si è già ricordato come Monti - per sua stessa ammissione avesse maturato l'idea di «leggere [...] i classici come 'classici della libertà'» dopo aver apprezzato le potenziali ricadute in campo educativo della tesi alfieriana di Piero, discussa a Torino nel 1922. Eppure, riconsiderando le posizioni pedagogiche avanzate dal docente in un importante saggio ultimato nell'estate del'21, Scuola classica e vita moderna, appare evidente che le premesse teoriche di tale indirizzo

Diogene Laerzio); ben rappresentati erano anche l'epica e la lirica arcaica, il teatro comico e tragico - e la storiografia (spec. Erodoto e Senofonte); il catalogo è accessibile all'indirizzo: http://www.centrogobetti.it (ultimo accesso 02.01.18).

66 A. Monti, "Congiure al chiaro giorno», $R L$ III (1924), n. 43, p. 173.

67 Cf. l'annuncio di pubblicazione riportato su $R L$ III (1924), n. 44, p. 178.

68 Vd. le considerazioni di Ersilia Alessandrone Perona in P. Gobetri, Carteggio, p. 371, n. 1.

69 Per i rapporti tra Gobetti e Monti, cf. N. Воввіо, Italia fedele, p. 135-155, con G. Tesio, Augusto Monti. Attualità di un uomo all'antica, Cuneo, 1980, p. 99-139. 
fossero già implicite nella sua riflessione precedente ${ }^{70}$. Nel saggio, infatti, Monti non si limitava a presentare la cultura classica come parte essenziale dell'eredità spirituale della nazione, ma la celebrava anche come fonte inesauribile di ammaestramenti per il presente:

A noi preme di insegnare [...] non solo come parlassero gli antichi, ma anche e più come pensassero e come agissero, [...] con il proposito, non più di trasferirci negli antichi e nell'antico, ma di scoprire, attraverso gli antichi, noi stessi, attraverso l'antico il presente ${ }^{71}$.

Questa “adesione alla realtà nazionale» - o, per dirla crocianamente, questo «ripensare il passato dal presente» - abbinava al canonico apprendimento della grammatica un'interpretazione più ampia delle fonti, che considerava le testimonianze antiche non più come ctermini $a d$ quem» ma come punto di partenza per riflettere sull'attualità, sulla base del principio che «non c'è problema della vita contemporanea che non si sia presentato agli antichi pressappoco sotto gli stessi aspetti con cui si presenta a noi». In questo senso, il motto «leggere i latini e i greci da Italiano e per Italianis andava declinato in una duplice direzione: da un lato, la storia moderna poteva offrire una chiave di lettura irrinunciabile alla comprensione del passato («non vi è nulla che aiuti meglio ad intendere la storia della Grecia, dal medio evo dorico ad Alessandro, che lo studio di certi periodi della storia d'Italia»); dall'altro, le pagine antiche si dimostravano capaci di illuminare con inedita freschezza i drammi del presente («non ho mai così profondamente sentito l'Epitaffio di Pericle in Tucidide come nel '15 e nel '16, quando morivano in guerra i primi dei miei scolari; non si è mai tanto pensato alla battaglia di Canne come dopo Novara e Caporettos) $)^{\mathbf{7 2}}$.

Tali considerazioni, elaborate quando Monti e Gobetti ancora non si conoscevano, riassumono perfettamente quell'ideale di stretta cintimità fra la vita nostra quotidiana ed il pensiero degli antichi» che avrebbe poi guidato - anche per iniziativa di Monti - il riuso polemico dei classici su La Rivoluzione Liberale.

70 Per un'efficace sintesi della pedagogia e della politica scolastica di Monti all'altezza dei primi anni Venti, vd. T. Tomasi, «Politica scolastica ed impegno educativo negli scritti di Augusto Monti», in G. Tesio (a cura di), Augusto Montinel centenario dellanascita, Torino, 1982, p. 9-22; A. D’Orsi, “La classicità dei moderni. Augusto Monti allievo di Giuseppe Fraccaroli', in A. Cavarzere-G.M. Varanini (a cura di), Giuseppe Fraccaroli (1849-1918). Letteratura, filologia e scuola fra Otto e Novecento, Trento, 2000, p. 59-75; G. Benedetto, "Rifar da capo: l'istruzione classica dopo l'Unità», in C.G. Lacaita-M. Fugazza (a cura di), L'istruzione secondaria nell'Italia unita (1861-1901), Milano, 2013, p. 83-87; G. Tognon, "Postfazione», in A. Monti, Scuola classica e vita moderna, Roma, 2016, p. 205-219.

71 A. Monti, Scuola classica e vita moderna, Torino, 1923, p. 20-21.

72 Ivi, p. 21-22, 59, 65-67. 
Non sorprenderà dunque che sia stato proprio Gobetti, entrato in contatto con il docente nel '21, ad accogliere con entusiasmo la proposta di pubblicare Scuola classica e vita moderna presso la propria casa editrice, annunciandone calorosamente l'uscita il 19 ottobre 1922:

È il testamento spirituale di un professore che ha dedicato venti anni di lavoro a vivere la scuola classica come fattore di modernità. Un libro che farà rimpiangere a molti di non essere stati a imparare greco e latino con A. Monti. Parlare poi delle doti di scrittore arguto e fine che vi si manifestano è superfluo per i lettori della Rivoluzione Liberale che bene le conoscono ${ }^{73}$.

Le parole di Piero sono importanti anche per un altro aspetto. Porre l'accento sull'educazione classica come "fattore di modernità) significava infatti funzionalizzare il modello di lettura proposto da Monti - e poi fatto proprio anche da La Rivoluzione Liberale - a uno degli obiettivi cardine della proposta politica gobettiana, che individuava proprio nell'approdo alla «modernità» - intesa come sviluppo armonico delle istituzioni liberal-democratiche - il fine ultimo dell'evoluzione dello stato. Nell'interpretazione di Gobetti, tale sviluppo sarebbe coinciso con il completamento del processo risorgimentale e avrebbe portato l'Italia a raggiungere standard politico-sociali finalmente degni degli stati europei più avanzati - su tutti, Francia e Inghilterra - i cui assetti istituzionali erano fondati sul rigoroso rispetto delle libertà individuali, sul protagonismo delle classi popolari e sul rifiuto di ogni autoritarismo ${ }^{74}$.

La parola dei classici finiva insomma, nella comune interpretazione di Gobetti e Monti, per alimentare un orizzonte programmatico del tutto estraneo a quello

73 Annuncio di pubblicazione apparso in $R L$ I (1922), n. 30, p. 114. Corsivo mio. Monti propose all'amico la stampa del libro il 2 ottobre 1922; pochi giorni dopo Gobetti mise in lavorazione il volume, che uscì nel marzo 1923, cf. P. Goветті, Carteggio, p. 339-340; G. Tognon, “Postfazione», in A. Monti, Scuola classica e vita moderna, Torino, 2016, p. 205-208; P. Gobetti, Carteggio 1923, a cura di E. Alessandrone Perona, Torino, 2017, passim.

74 Si rilegga, al proposito, il profilo di 'governo ideale' abbozzato in P. Gobetti, “La successione», p. 185, già ricordato supra (p. 60-61): “Le opposizioni devono superare il punto morto dell'attuale vita italiana dichiarandosi pronte alla successione. [...] Un governo così composto [...] sarà un governo di partiti responsabili e non di avventurieri e di dittatori; sarà il primo governo che potrà conservare l'ordine, perché parlerà col prestigio della democrazia, del consenso e di una parte delle classi proletarie; invece che da un blocco di interessi personali, nascerà da una collaborazione leale e aperta di forze e di programmi diversi, ma non contradditori, controllati dagli istituti democratici moderni; invece di essere uno Stato balcanico o sud-americano l'Italia si metterà sulla via di diventare uno Stato europeo moderno». Corsivi e sotto lineati miei. 
proposto dal classicismo fascista. Quest'ultimo, come ha ben chiarito Luciano Canfora, celebrava l'Italia mussoliniana come legittima erede dell'impero romano per rinfocolare propagandisticamente l'antagonismo nei confronti del «mondo moderno", che veniva identificato con ogni forma di ordine politico favorevole a un'evoluzione in senso liberale o socialista degli ordinamenti statali ${ }^{75}$. Ma c'è di più. Sulla base di questa opposta valutazione del processo di «modernizzazione» della penisola, classicismo gobettiano e classicismo fascista si facevano portatori di due visioni nettamente antitetiche della storia d'Italia. Da un lato, la propaganda mussoliniana, ricercando nel passato italiano un modello politico da far rivivere nel XX secolo, esprimeva un approccio continuista, che individuava nella Roma imperiale un paradigma statuale virtuoso, valido per il presente ${ }^{76}$. Il classicismo gobettiano, al contrario, predicava una netta discontinuità rispetto alle esperienze storiche precedenti, considerandole del tutto inadeguate all'Italia contemporanea. La valorizzazione della tradizione classica si doveva piuttosto concretizzare in un'accurata selezione di auctoritates - «i classici della libertà», appunto che avevano il compito di stimolare gli italiani a ripudiare i vizi storici del loro passato (servilismo cortigiano; reazionarismo cattolico; parassitismo economico), indirizzandoli verso un modello di statualità del tutto nuovo, capace di portare a termine il processo risorgimentale che si era arenato con l'unità politica della penisola.

Se proviamo dunque a riconsiderare il corsivo tucidideo da questa prospettiva, ci accorgiamo che alle spalle di un esperimento di lotta politica provocatorio e spregiudicato si delineava un approccio alla tradizione estremamente complesso, che esprimeva a più livelli - storico, morale e antropologico - una forma di radicale rifiuto della Weltanschauung fascista. Com'era lecito attendersi, tale prospettiva venne progressivamente soffocata insieme alle iniziative di Gobetti, le quali, complice la prematura scomparsa di Piero nel febbraio 1926, si interruppero bruscamente (La Rivoluzione Liberale chiuse nell'autunno del '25; Il Baretti nel '28). Eppure, nonostante queste battute d'arresto, la linea gobettiana di interpretazione dei classici non si esaurì, riemergendo carsicamente negli scritti e nei ricordi di sodali e collaboratori: da Mario Fubini - i cui lavori alfieriani si dimostrarono sempre memori della lezione di Gobetti ${ }^{77}$ - ad Augusto Monti, che riservò le ultime pagine del suo testamento pedagogico - I miei conti con la scuola (1965) - a un'appendice significativamente intitolata La rivoluzione liberale e la scuola di domani ${ }^{78}$.

75

76 Vd. ancora L. CANFora, Ideologie, p. 76-132 e iD., Le vie, p. 244-277.

77 Cf. A. FABrizi, Che ho a che fare, p. 7-13.

78 A. Monti, I miei conti con la scuola. Cronaca scolastica italiana del secolo XX, Torino, 1965, p. 361ss. 
In questo quadro, anche la voce di Tucidide tornò a farsi sentire nelle riflessioni di un altro gobettiano, Luigi Salvatorelli, che il 10 dicembre 1944, pochi mesi prima della fine della guerra, pubblicò su La Nuova Europa un lungo articolo su “Presente e avvenire d'Europa». In esso, Salvatorelli discuteva i caratteri dello "statalismo totalitario», descrivendo il "capovolgimento di tutti i valori» che aveva contraddistinto il fascismo con toni e concetti direttamente ricavati dalla stessa sezione delle Storie - quella dedicata alla stasis corcirese (spec. Th. III, 82, 4-5) - già centonata vent'anni prima ${ }^{79}$. Questa precisa reminiscenza, che confermava l'integrabilità di Tucidide nell'orizzonte del liberalismo gobettiano, non passò inosservata all'occhio attento di Alfredo Rizzo, il quale rilevò, in uno scritto pubblicato su Studium nel 1945, quanto le parole salvatorelliane dessero cuna chiara ed acuta analisi del male che ci oppresse», esprimendo “l'eco d'una voce antica che risonava con tono tanto moderno [...] e che parlava eloquentemente dell'attualità di Tucidide ${ }^{80}$ ).

Anche a molti anni di distanza, insomma, il classicismo coltivato nella cerchia di Gobetti si faceva portatore di una visione ampia e attualizzante della tradizione, che diveniva parte essenziale di un modo di intendere la politica - caratteristicamente gobettiano - fondato sull'idea che nulla si poteva cambiare nella vita di una nazione “se prima non si trasformavano le menti e i caratteri» dei suoi cittadini ${ }^{81}$. Questa saldatura organica tra militanza politica e attività culturale costituì, nel parere di Norberto Bobbio, il «nucleo» stesso del “gobettismo» e si pose all'origine di quel particolare legame tra Piero e la sua cerchia, che durò ben oltre gli anni della dittatura, sopravvivendole proprio grazie all'cinsegnamento di vita» offerto da Gobetti ai suoi “adepti» ${ }^{82}$. Un lascito che lo stesso Monti, in un commosso ricordo del giovane amico, volle descrivere attraverso l'ennesimo riferimento classico, paragonando il atesoro di pensieri e di norme» ereditato da Piero alla perfetta costituzione che il «nomoteta» Licurgo aveva affidato agli Spartani, pregandoli di non modificarla fino a quando egli non avesse fatto ritorno da un lungo viaggio, dal quale tuttavia il legislatore non sarebbe più rientrato:

Quando era stata [...] la trigesima della morte del loro Piero, si eran trovati i fedelissimi torinesi nel quartierino di via Fabro caldo ancora della sua presenza:

79 L. Salvatorelli, “Presente e avvenire d'Europa», La Nuova Europa I (1944), n. 1, p. 1: “La parvenza fu messa al posto della realtà, il male fu proclamato bene: la codardia delinquente si disse eroismo, l'illimitato arbitrio diritto, la tirannide poliziesca libertà vera, il cupido e fraudolento arricchimento disinteresse e sacrificio, il tradimento della patria patriottismo geniale. Fu il capovolgimento di tutti i criteri, di tutti i valori».

80 A. Rizzo, "Ritorno di Tucidide», Studium 41 (1945), ora in ID., Scienza impura. Pagine di filologia e umanità, Roma, 1981, p. 161-162.

81 N. Воввіо, Italia fedele, p. 130-131.

82 Ivi, p. 123-124, 130-131. 
era toccato a me dir due parole, ero fresco tuttavia del latino e del greco insegnato altrove per tanti anni, mi scapparon fuori le reminiscenze classiche: ricordai il legislatore di Sparta, il nomoteta che, fissata la costituzione della sua patria, era partito per «un lungo viaggio» raccomandando di nulla mutare di quello statuto finché lui non fosse tornato; e non era tornato più. Noi non avevamo visto morire Piero Gobetti: per noi era solamente partito lasciandoci un tesoro di pensieri, di norme, uno "statuto", che a noi toccava serbar intatto finché il "nomoteta» non fosse tornato. [...] Da un pezzo in qua io amo figurarmi a volte il nomoteta ritornato fra noi a far ispezione, come i (fedeli» abbian tenuto consegna. E che sia soddisfatto, tuttassieme. È stata montata bene la guardia al suo «statuto», ed è esso ancora, nonostante tutto, che governa il meglio della vita pubblica italiana ${ }^{83}$.

\author{
Luca lori \\ Università di Parma \\ Dipartimento DUSIC \\ Unità di Storia \\ Strada Massimo D’Azeglio 85 \\ 43125 Parma, Italia \\ luca.iori@unipr.it
}

\title{
Appendice
}

Di seguito si fornisce la trascrizione dell'articolo “Tucidide e il fascismo», pubblicato in La Rivoluzione Liberale, anno III, n. 43 (18 novembre 1924). Per ogni excerptum si rimanda, tra parentesi quadre, al capitolo dell'opera tucididea dal quale il passo è tratto.

\section{TUCIDIDE E IL FASCISMO}

\section{La Marcia su Roma e i salvatori della Patria}

«Fu verso quell'epoca che la democrazia venne abolita in Atene...». [Th. VIII, 63, 3] «Da più tempo gli oligarchi avevano fatto circolare la voce che tutti i diritti spettavano unicamente agli uomini di guerra ed ai soli cittadini capaci di servire la città con la persona e con gli averi. Non si trattava in realtà che di un tranello teso alla moltitudine, poiché era chiaro che soltanto i fautori del colpo di Stato si sarebbero avvantaggiati del potere». [Th. VIII, 65, 3 - 66, 1]

83 A. Monti, “Con Piero Gobetti», p. 208. 
Gli assassini

«Si principiò con l'assassinio di Androclo, uno dei capi più in vista della democrazia. Quindi tutta la parte popolare fu presa d'assalto con un crescendo di uccisioni sistematiche». [Th. VIII, 65, 2]

\section{La paura}

“Così numerosa ed insolente era divenuta la fazione oligarchica, che non si trovava alcuno che osasse alzare la voce contro di essa. Se, per caso, qualche temerario l'avesse tentato, le più sanguinose vendette erano pronte a colpirlo». [Th. VIII, $66,2]$

\section{L’impunità degli assassini}

“Lo Stato non si curava di ricercare i colpevoli di tanti misfatti. Piena impunità era accordata agli assassini, che, anche se conosciuti, potevano circolare liberamente...). [Th. VIII, 66, 2]

\section{Il silenzio}

“Il popolo non osava protestare. Egli vivea in tale stato di spavento, che si riputava già felice di poter sfuggire con il silenzio allo sterminio che lo minacciava». [Th. VIII, 66, 2]

\section{I traditori}

“Così, malgrado lo sdegno del quale tutti erano accesi, non si faceva un passo per organizzare una difesa. Ogni coraggio era prostrato. Un'aura di terrore travolgeva ogni cosa. Si credevano anche gli oligarchi in maggior quantità o più potenti di quello che, in realtà, non lo fossero. Aggiungi che non si era sicuri di nessuno, dappoichè uomini che non si sarebbero mai sospettati capaci di tradire il popolo, erano passati alla oligarchia, la cui forza principale riposava appunto su questi traditori». [Th. VIII, 66, $4+$ Th. VIII, 66, $3+$ Th. VIII, 66, 5]

\section{L'insegnamento della guerra}

«Nella pace e nella prosperità, la città ed i privati sono meglio e più saggiamente inclinati, perché non conoscono le dure necessità; ma la guerra, distruggendo ogni benessere, porge continue lezioni di violenza e rende l'indole dei cittadini conforme all'asprezza dei tempi. Ardeva la guerra civile nelle città, e quelle ultime che sorgevano in armi si studiavano di sorpassare le prime nel trovare nuovi modi di aggressione ed inusitati supplizi». [Th. III, 82, 2-3]

\section{La lingua nuova}

«Era cambiato il consueto significato dei vocaboli. La sconsigliata audacia si chiamava coraggio, il cauto indugio timidezza, la moderazione viltà. Sicuro era considerato solo l'uomo violento, il sospetto circondava gli egregi cittadini». [Th. III, 82, 4-5] 


\section{Il ramoscello di ulivo}

“La fiducia scambievole non si fondava sulla religione, ma sulla complicità dei misfatti; le oneste profferte della parte contraria non si accettavano in buona fede, bensì quando si scorgesse che si resterebbe superiori ad accettarle». [Th. III, 82, 6-7]

“La semplicità, dote principale di un’anima nobile, derisa, sparì; prevalse il ridurre le menti in reciproca gara di diffidenza; non più sicurezza di parole, non più timore di giuramento; sicché trovando ovunque più forti ragioni di non aver fiducia, l'uomo meditava piuttosto il modo di non essere offeso, che indursi a fidarsi di chicchessia». [Th. III, 83, 1-2]

\section{Inflessibilmente}

«Di tutti questi mali era cagione la sete del comando, che da ambizione e da cupidigia procede. Queste passioni travolgevano gli spiriti e li eccitavano a osare qualunque scelleratezza». [Th. III, 82, 8]

\section{I "Ras" e l'amministrazione}

“Nelle città i capi delle fazioni, con il pretesto di un regime di perfetta uguaglianza gli uni, e un discreto reggimento di pochi gli altri, aiutavano la cosa pubblica di nome, e in fatto la riducevano in isfacelo. Perciò, studiando a scalzarsi l'un l'altro, osavano e compivano le più orribili cose, aggravando le pene, non secondo la giustizia e il vantaggio della repubblica, ma secondo che le determinava il loro capricciom. [Th. III, 82, 8]

\section{Discordie tra $i$ "Ras"}

“Non esitavano a soddisfare le rispettive cupidigie, sia con il condannare altrui con ingiusto suffragio, sia col procacciarsi armata mano superiorità, di maniera che ambedue le fazioni non avevano alcun riguardo alla morale; ma quelli cui accadesse, con speciosità di parole, di fare un bel colpo, erano i più reputati; dove i cittadini che tenevano la via di mezzo fra entrambe le parti, venivano nondimeno perseguitati, o per non aver dato mano ad una, o per invidia di vederli fuori del tafferuglio». [Th. III, 82, 8]

Tucidide : lib. VIII, 43, 45, 46 [l. 63, 65, 66]; lib. III, 82-83. 
